\title{
Cystamine and cysteamine increase brain levels of BDNF in Huntington disease via HSJ1b and transglutaminase
}

\begin{abstract}
Maria Borrell-Pagès, ${ }^{1}$ Josep M. Canals, ${ }^{2}$ Fabrice P. Cordelières, ${ }^{1}$ J. Alex Parker, ${ }^{3}$ José R. Pineda, $^{2}$ Ghislaine Grange, ${ }^{1}$ Elzbieta A. Bryson, ${ }^{1}$ Martine Guillermier, ${ }^{4}$ Etienne Hirsch, ${ }^{5}$ Philippe Hantraye,${ }^{4}$ Michael E. Cheetham, ${ }^{6}$ Christian Néri, ${ }^{3}$ Jordi Alberch, ${ }^{2}$ Emmanuel Brouillet, ${ }^{4}$
\end{abstract}

Frédéric Saudou, ${ }^{1}$ and Sandrine Humbert ${ }^{1}$

\begin{abstract}
${ }^{1}$ Institut Curie, CNRS UMR 146, Orsay, France. 2Departament de Biologia Cellular i Anatomia Patològica, Facultat de Medicina, Institut d'Investigacions Biomèdiques, Universitat de Barcelona, Barcelona, Spain. ${ }^{3}$ INSERM, Avenir Group, Laboratory of Genomic Biology, Centre Paul Broca, Paris, France. ${ }^{4}$ URA CEA/CNRS 2210, Service Hospitalier Frédéric Joliot and ImaGene program, Département de Recherches Médicales, Direction des Sciences du Vivant, CEA, Orsay, France. 5INSERM U679, Neurologie et Thérapeutique Expérimentale, Hôpital de la Salpêtrière, Paris, France. ${ }^{6}$ Division of Molecular and Cellular Neuroscience, Institute of Ophthalmology, University College London, London, United Kingdom.
\end{abstract}

\begin{abstract}
There is no treatment for the neurodegenerative disorder Huntington disease (HD). Cystamine is a candidate drug; however, the mechanisms by which it operates remain unclear. We show here that cystamine increases levels of the heat shock DnaJ-containing protein 1b (HSJ1b) that are low in HD patients. HSJ1b inhibits polyQ-huntingtin-induced death of striatal neurons and neuronal dysfunction in Caenorbabditis elegans. This neuroprotective effect involves stimulation of the secretory pathway through formation of clathrin-coated vesicles containing brain-derived neurotrophic factor (BDNF). Cystamine increases BDNF secretion from the Golgi region that is blocked by reducing HSJ1b levels or by overexpressing transglutaminase. We demonstrate that cysteamine, the FDA-approved reduced form of cystamine, is neuroprotective in HD mice by increasing BDNF levels in brain. Finally, cysteamine increases serum levels of BDNF in mouse and primate models of HD. Therefore, cysteamine is a potential treatment for $\mathrm{HD}$, and serum BDNF levels can be used as a biomarker for drug efficacy.
\end{abstract}

\section{Introduction}

Huntington disease (HD) is a devastating neurodegenerative disorder characterized by involuntary abnormal movements, personality changes, and dementia (1). The dominantly inherited causal gene encodes the huntingtin protein, which contains an abnormal polyglutamine (polyQ) expansion in HD patients. HD develops when the expansion exceeds 35 glutamine residues, and there is a strong inverse correlation between the number of residues and the age at onset. HD is characterized by the preferential dysfunction and death of striatal neurons in the brain and the presence of neuritic and intranuclear inclusions in neurons (2).

There is currently no effective treatment to prevent or delay disease progression, and death usually occurs within 10-20 years after the appearance of the first clinical symptoms. One of the most promising candidate drugs for HD is cystamine, a compound described as an in vitro transglutaminase (TGase) inhibitor. TGase is a calcium-dependent enzyme that catalyzes the formation of $\varepsilon^{-}(\gamma$-glutamyl)lysine isopeptide bonds between a polypeptide-bound glutamine and a lysine of the protein substrate $(3,4)$. TGase is suspected of participating in HD pathogenesis (5). PolyQ-containing peptides and polyQ-huntingtin are substrates for TGase $(6,7)$. Moreover, TGase is upregulated in the brains of HD patients and of HD mice (8-11).

Nonstandard abbreviations used: BDNF, brain-derived neurotrophic factor; BFA, brefeldin A; CCV, clathrin-coated vesicle; HD, Huntington disease; hsc70, heat shock cognate $70 \mathrm{kDa}$; HSJ1, heat shock DnaJ-containing protein $1 \mathrm{~b}$; Hsp, heat shock protein; NII, neuronal intranuclear inclusion; 3NP, 3-nitropropionic acid; polyQ, polyglutamine; TGase, transglutaminase; TGase 2, tissue TGase 2; TGN, trans-Golgi network. Conflict of interest: The authors have declared that no conflict of interest exists. Citation for this article: J. Clin. Invest. 116:1410-1424 (2006). doi:10.1172/JCI27607.
Given their enzymatic properties, TGases might promote aggregate formation in HD. The observations that TGase is recruited into neuronal intranuclear inclusions (NIIs) $(10,12)$ and that NIIs are reduced in a cystamine-treated mouse model of HD (10) are consistent with this idea. However, cystamine treatment of HD mice does not necessarily result in fewer NIIs (11), and an increase in NIIs is observed in HD mice that are deficient for 1 of the TGase isoenzymes, tissue TGase 2 (TGase 2) $(13,14)$. Nevertheless, both HD mice lacking TGase 2 and HD mice treated with cystamine have less neuronal death, improved motor performance, and prolonged survival $(10,11,13-15)$. In addition to its role as an in vitro inhibitor of TGase, cystamine could act through other mechanisms $(14,16,17)$. It inhibits caspase 3 activity and increases the levels of glutathione in cell models (18). It also increases the level of L-cysteine in cell models and animals $(17,19)$.

To understand better the beneficial effects of cystamine, Karpuj and colleagues treated HD mice with cystamine and analyzed large-scale profiles of transcription in the brain, where they identified genes whose transcription was specifically influenced by cystamine treatment (11). In particular, transcripts of DnaJ-type heat shock proteins (Hsps) were elevated upon cystamine treatment (11). Numerous other studies have highlighted the important role of Hsps in HD and in other polyQ disorders in cellular and animal models (for reviews, see refs. 20-22). Hsps are of particular relevance to HD not only because they prevent aggregation of mutant proteins and solubilize aggregated proteins but also because they promote ubiquitination and degradation of abnormal proteins and suppress the antiapoptotic program $(22,23)$. Cystamine might, therefore, be protective in HD by increasing the transcription of Hsps that, in turn, protect cells against polyQ-huntingtin-induced toxicity. 
A

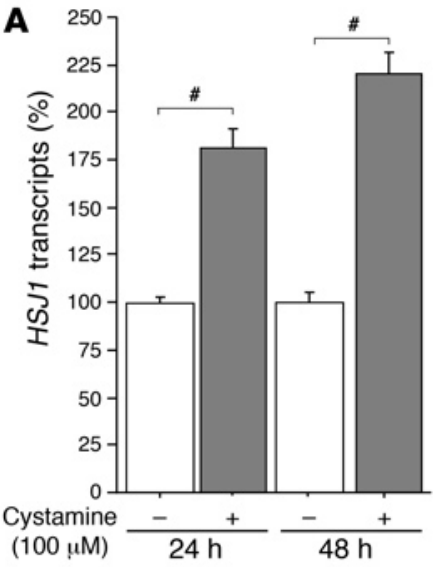

B

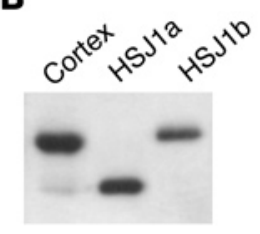

C

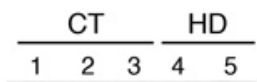

HSJ1b

$\beta$-Actin

D

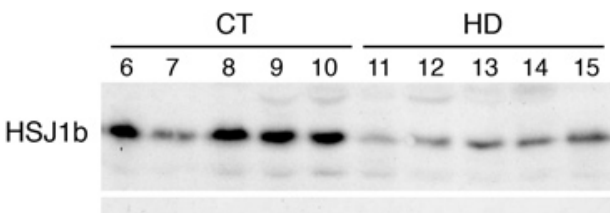

$\beta$-Actin

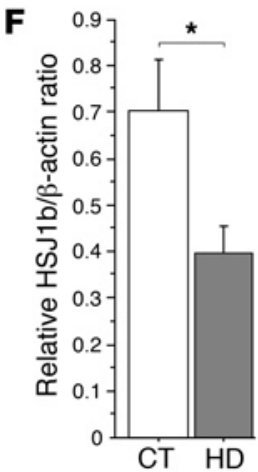

E

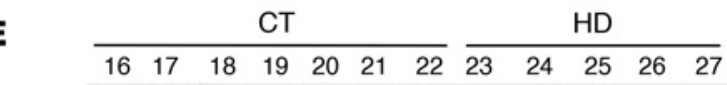

HSJ1b

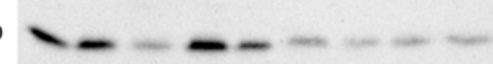

$\beta$-Actin

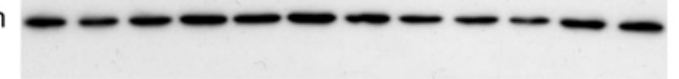

\section{Figure 1}

Cystamine increases HSJ1 transcript levels in neuronal cells, while HSJ1b is decreased in postmortem brain extracts from HD patients. (A) Data revealed a statistically significant increase in HSJ1 transcripts induced by cystamine treatment in comparison to control at 24 hours (Student's $t$ test, $t_{[21]}=5.77 ; P<0.0001$ ) and at 48 hours (Student's $t$ test, $t_{[10]}=9.88$; $P<0.0001)$. (B) Protein extracts prepared from 1 control human cortical postmortem sample and from HEK 293T cells transfected with HSJ1a or HSJ1b were immunoblotted with an anti-HSJ1 antibody. The major brain isoform of HSJ1 proteins was the HSJ1b isoform. (C-E) Protein extracts were prepared from whole striatum (C), putamen (D), and caudate nucleus (E) of control (CT) and HD individuals and analyzed as in B. Immunoblotting with an anti- $\beta$-actin antibody was used as a control. (F) Quantification of the Western blots presented in $\mathbf{C}-\mathbf{E}$ showed a statistically significant decrease in the protein level of HSJ1b in HD samples $(n=12)$ compared with control samples $(n=15)$ (Student's $t$ test, $\left.t_{[25]}=2.33 ; P=0.028\right)$. ${ }^{*} P<0.05,{ }^{\#} P<0.0001$.
To study the protective mechanism elicited by cystamine, we focused on the Homo sapiens heat shock DnaJ-containing protein $1 \mathrm{~b}$ (HSJ1b), which is enriched in the brain. We report that HSJ1b and cystamine are neuroprotective in HD by increasing the release of brain-derived neurotrophic factor (BDNF) - a trophic factor that is depleted in HD brains and is crucial for the survival of striatal neurons (24-27) - from neurons both in culture and in animals. We demonstrate that cystamine positively regulates BDNF release not only by increasing HSJ1 transcripts but also by inhibiting TGase. We next show that cysteamine, the FDA-approved reduced form of cystamine, is neuroprotective in HD mice by enhancing BDNF levels in brain. Indeed, changes in endogenous BDNF levels modulate the cystamine-induced neuroprotective effect in HD mice. Finally, BDNF blood levels are low in mice and primate models of HD and can be increased by injection of cysteamine. Our study provides not only new mechanisms by which cystamine promotes neuroprotection in HD, but also a potential new treatment for HD using its FDA-approved reduced form, cysteamine.

\section{Results}

HSJ1 transcripts are elevated upon cystamine treatment. To confirm that cystamine treatment leads to an increase in the amount of HSJ1 transcripts, we treated mouse immortalized neuronal cells (28) with $100 \mu \mathrm{M}$ cystamine for 24 or 48 hours, lysed the cells, isolated mRNAs, and performed real-time quantitative RT-PCR (real-time RT-PCR) using validated oligonucleotides (Figure 1A). We observed a statistically significant increase in HSJ1 transcripts of 1.7-fold after 24 hours treatment and of 2.2-fold after 48 hours (Figure 1A). Thus, HSJ1 transcripts are elevated upon cystamine treatment of both neuronal cells and the R6/2 mouse model of HD (11).
$H S J 1 b$ - the predominant HSJ1 isoform in brain - is decreased in HD brains. The HSJ1 gene generates, by alternate splicing, 2 proteins, HSJ1a and HSJ1b, that differ in their C termini. HSJ1 mRNAs are enriched in brain, and HSJ1a and HSJ1b proteins are found in various regions such as cortex, cerebellum, striatum, and retina, with HSJ1b more abundant than HSJ1a (Figure 1, B-E) (29). To analyze the relevance of HSJ1 proteins in the context of HD, we determined their levels in postmortem samples of striatum from HD patients. Striatum that includes caudate nucleus and putamen is the most severely affected region in HD (1). By immunoblotting the samples with an antibody against HSJ1, we found a dramatic decrease in the level of HSJ1b protein in HD patients compared with control individuals (Figure 1, C-E). Anti- $\beta$-actin was used as a control for protein loading. HSJ1a was expressed at low or undetectable levels in both control and HD brain samples. We quantified the downregulation of HSJ1b and found it to be statistically significant (Figure 1F). These results show that the levels of HSJ1b, the main isoform of HSJ1 in brain, are reduced in the pathological situation and are of further support for a role of HSJ1b in HD pathogenesis. The postmortem samples represent late stages of the disease. Therefore, such a decrease could be attributed to the selective death of neurons expressing HSJ1b. However, these brain extracts show no profound modifications in the levels of calbindin, a specific marker of medium spiny neurons (data not shown). This suggests that the decreased levels of HSJ1b in postmortem samples are not merely a reflection of cell death.

HSJ1 proteins protect striatal neurons from polyQ-buntingtin-induced cell death. We next investigated whether HSJ1 proteins possess neuroprotective properties by studying a neuronal model of HD that recapitulates the main features of the disease (24). We transfected 

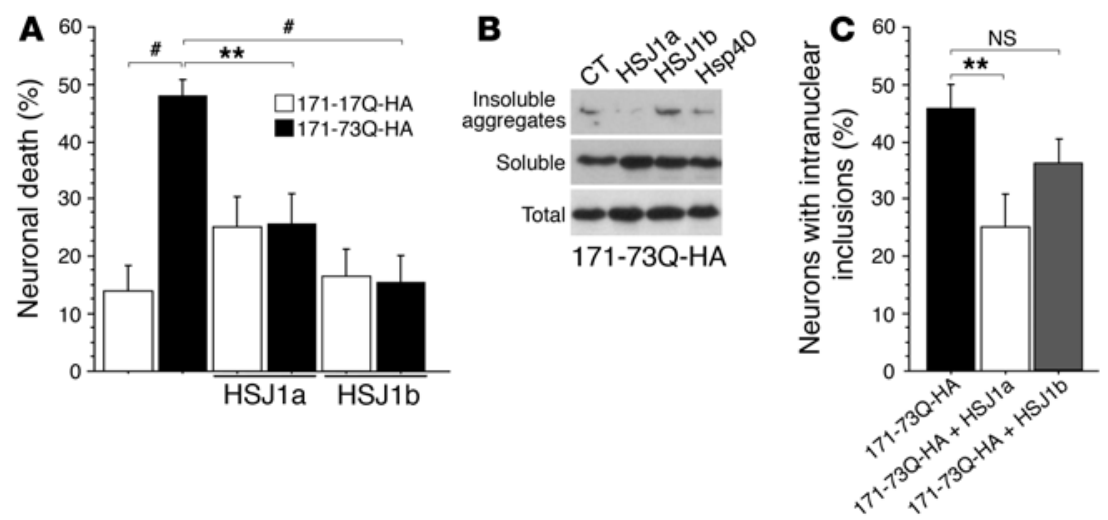
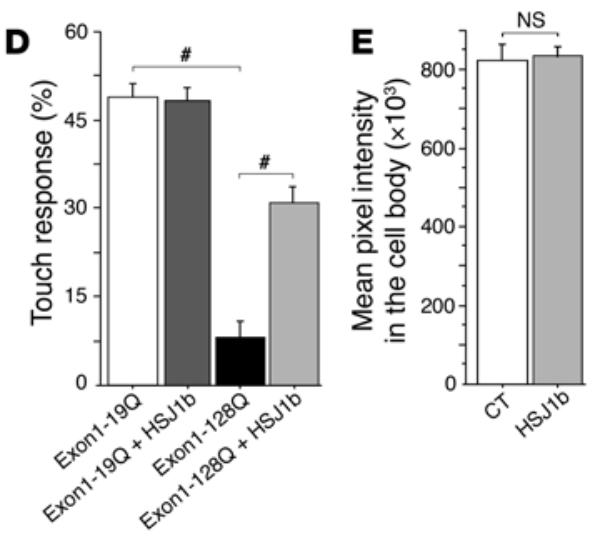

Figure 2

HSJ1 proteins and polyQ-huntingtin-induced toxicity and dysfunction. (A) Striatal neurons were transfected with 171-17Q-HA or 171-73Q-HA and HSJ1a, HSJ1b, or the corresponding empty vectors. Data (ANOVA, $F_{5,40}=6.89 ; P<0.0001$ ) demonstrated that cell death was significantly increased by 171-73Q-HA construct (post-hoc Fisher's test, $P<0.01$ ) and blocked by cotransfection with HSJ1a (post-hoc Fisher's test, $P=0.0043$ ) or HSJ1b (post-hoc Fisher's test, $P<0.0001$ ). (B) Cell extracts prepared from 171-73Q-HA-transfected HEK 293T cells were analyzed by immunoblotting using an anti-HA antibody. (C) Striatal neurons were transfected with 171-17Q-HA or 171-73Q-HA together with HSJ1a, HSJ1b, or the corresponding empty vectors. Data (ANOVA, $F_{2,15}=4.43 ; P=0.031$ ) revealed a statistically significant decrease in the percentage of neurons with intranuclear inclusions in the presence of HSJ1a (post-hoc Fisher's test, $P=0.0094$ ) but not of HSJ1b (NS). (D) Data (ANOVA, $F_{10,87}=23.44 ; P<0.0001$ ) revealed a statistically significant decrease in mechanosensation of touch receptor neurons in the tail of animals expressing the exon 1-128Q-GFP construct compared with neurons expressing exon 1-19Q-GFP (Student's $t$ test, $t_{[16]}=16$. 12; $P<0.0001$ ). Loss of touch response mediated by exon 1-128Q-GFP was inhibited by expression of HSJ1b (Student's $t$ test, $t_{[16]}=9.01$; $P<0.0001$ ). (E) Morphometric analysis revealed no change in the aggregation of fusion proteins in the cell bodies of neurons from HSJ1bexpressing animals (Student's $t$ test, $t_{[198]}=1.22 ; \mathrm{NS}$ ). ${ }^{* \star} P<0.01,{ }^{\#} P<0.001$.

primary cultures of striatal neurons with constructs encoding the first 171 amino acids of huntingtin with 17 (wild-type, 171-17QHA) or 73 glutamines (mutant, polyQ, 171-73Q-HA), either alone or in the presence of a construct expressing HSJ1a or HSJ1b, and analyzed neuronal death 24 hours after transfection (Figure 2A). As expected, the 171-73Q-HA construct induced a statistically significant increase in neuronal death compared with the 171-17QHA construct. Interestingly, HSJ1a and HSJ1b decreased neuronal death induced by the 171-73Q-HA fragment of huntingtin. These findings show that HSJ1 proteins exert a neuroprotective effect on polyQ-huntingtin-induced neuronal death.

HSJ1 a but not HSJ1b reduces intranuclear inclusions. Hsps are known to assist in the refolding of misfolded proteins and/or in the transfer of such proteins to the proteasome for degradation $(23,30)$. Therefore, most Hsps reduce the aggregates in various HD models (22). Using an aggregation assay (31), we analyzed the effect of HSJ1 proteins on the formation of insoluble aggregates induced by the 171-73Q-HA fragment of huntingtin (Figure 2B). The DnaJtype Hsp40 (HDJ2/HSDJ) was used as a positive control capable of solubilizing aggregates $(20,22)$. Surprisingly, HSJ1a and HSJ1b elicited different responses in this assay. Whereas HSJ1a reduced insoluble aggregates in a comparable manner to Hsp40, HSJ1b had no effect on the solubility of polyQ-huntingtin-induced aggregates. To confirm this differential response in a more physiological situation, we transfected primary cultures of striatal neurons with 171-73Q-HA and HSJ1a, HSJ1b, or the corresponding empty vector and scored the transfected neurons for the presence of NIIs (Figure 2C). Again, we found that whereas HSJ1a significantly reduced the formation of NIIs, HSJ1b had only a weak effect.

These findings suggest that HSJ1a acts as a "typical" chaperone that unfolds misfolded proteins, whereas HSJ1b has a beneficial effect on polyQ-huntingtin-induced neuronal death independent of polyQ aggregation. As HSJ1a is almost undetectable in brain (Figure 1), we focused our study on the predominant isoform, HSJ1b.

$H S J 1 b$ rescues mutant polyQ dysfunction in Caenorbabditis elegans neurons. To test whether HSJ1b may protect against the early phases of polyQ-huntingtin-induced neuronal dysfunction in vivo, we investigated the effects of HSJ1b in C. elegans transgenics that express an exon 1-like N-terminal fragment of huntingtin fused to GFP in their touch receptor neurons. This animal model of neuronal cell response to polyQ-expanded huntingtin shows polyQ-dependent neuronal dysfunction without cell death (32, 33). We generated transgenic strains of nematodes that express human HSJ1b in touch receptor neurons. Four independent stable lines were crossed with lines stably expressing a GFP-fused exon 1-like $\mathrm{N}$-terminal fragment of huntingtin with 19 or 128 glutamines (exon 1-19Q-GFP or exon 1-28Q-GFP). Quantitative RT-PCR and Western blotting revealed no effect of HSJ1b on huntingtin transgene expression and protein levels (Supplemental Figure 1; supplemental material available online with this article; doi:10.1172/JCI27607DS1). We analyzed the touch response of the mutant polyQ and control worms in the absence or presence of HSJ1b. As previously reported $(32,33)$, animals expressing exon 1-128Q-GFP presented a statistically significant decrease in response to light touch at the tail compared with the exon 1-19Q-GFP-expressing worms (Figure 2D). Importantly, the loss of touch response induced by exon 1-128Q-GFP was inhibited by HSJ1b expression. In contrast, no statistical difference in cell body aggregation, measured as mean pixel intensity, was observed between exon 1-128Q-GFP strains and those with HSJ1b coexpression (Figure $2 \mathrm{E}$ ). We conclude that HSJ1b has neuroprotective effects in vitro by inhibiting striatal neuronal death and in vivo by reducing neuronal cell dysfunction without any major effects on polyQ-huntingtin aggregation. 

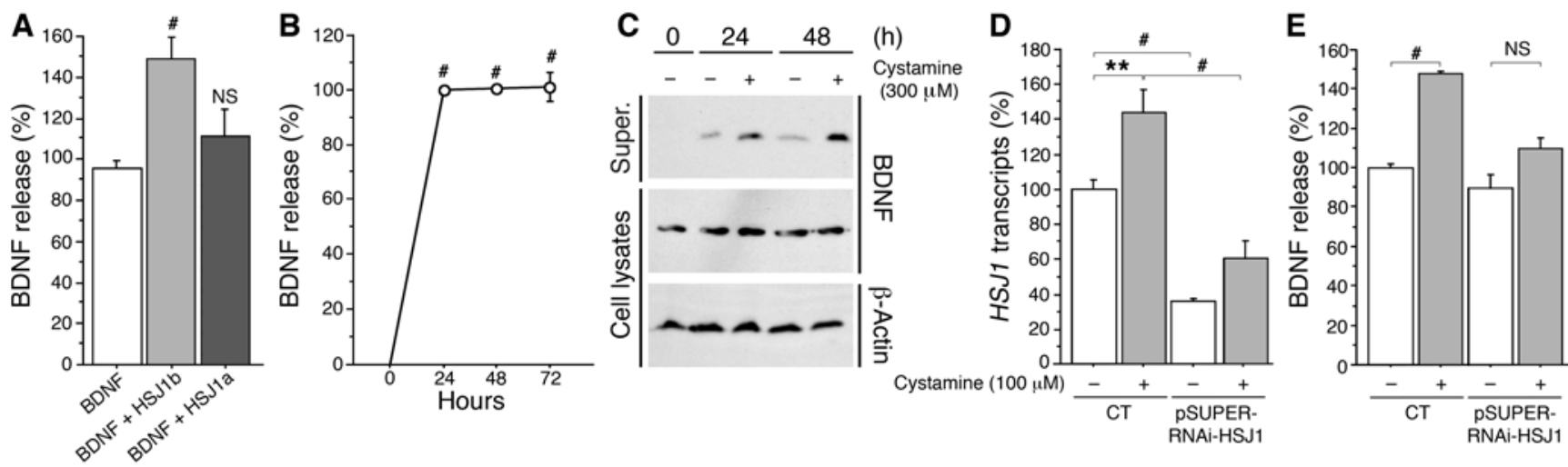

Figure 3

HSJ1b and cystamine increase BDNF release. (A) Neuronal cells were transfected with BDNF, HSJ1a, HSJ1b, or the corresponding empty vectors. Forty-eight hours after transfection, cells were washed with PBS and incubated for 30 minutes with DMEM, and supernatants (super.) were collected. Data (ANOVA, $F_{2,31}=9.17 ; P=0.0007$ ) revealed that HSJ1b (post-hoc Fisher's test, $P=0.0002$ ), but not HSJ1a (NS), induced a statistically significant increase in BDNF release. (B) Data (ANOVA, $F_{3,39}=323.66 ; P<0.0001$ ) revealed a statistically significant increase in BDNF content in the supernatant of $100 \mu \mathrm{M}$ cystamine-treated cells at 24,48 , and 72 hours (post-hoc Fisher's test, $P<0.0001$ ). (C) Cells transfected with BDNF and treated with cystamine were analyzed by immunoblotting with anti-BDNF and anti- $\beta$-actin antibodies. (D) Data (ANOVA, $\left.F_{3,29}=26.01 ; P<0.0001\right)$ revealed a statistically significant decrease in HSJ1 transcripts in cells transfected with siRNA-HSJ1 compared with control cells with or without cystamine treatment (post-hoc Fisher's test, $P<0.0001$ ). Cystamine increased HSJ1 transcripts in control conditions (post-hoc Fisher's test, $P=0.002$ ) but not in the presence of siRNA-HSJ1 (NS). (E) Cystamine did not increase BDNF release when HSJ1b levels were lowered by RNAi-HSJ1 (ANOVA, $F_{3,19}=7.59 ; P=0.0016$ ). Cells were cotransfected with BDNF and pSUPER-RNAi-HSJ1 and treated with cystamine 48 hours after transfection. There was a significant increase in BDNF release in cystamine-treated cells compared with control cells (post-hoc Fisher's test, $P=0.0005)$. ${ }^{* *} P<0.01,{ }^{\#} P<0.001$.

$H S J 1 b$ and cystamine promote BDNF release in neuronal cells. How does HSJ1b promote neuronal survival? Little is known about the function of HSJ1 proteins. We do know, however, that they interact with the Hsp70 family of proteins and stimulate their ATPase activity to regulate their substrate-binding capacities (34). Also, HSJ1 proteins inhibit the heat shock cognate $70 \mathrm{kDa}$ (hsc70) protein. Since hsc70 is a constitutive Hsp70 that removes clathrin from clathrin-coated vesicles (CCVs), we hypothesized that HSJ1 proteins might play a role in regulating intracellular trafficking of CCVs (35). Indeed, huntingtin itself is implicated in clathrinmediated endocytosis; it interacts with several proteins involved in this process, including HIP1, HIP12, PACSIN1, SH3GL3, and HIP14 (for a review, see ref. 36), and we recently demonstrated that huntingtin acts as a processivity factor to promote microtubule-based intracellular transport of BDNF-containing vesicles (27). In addition, the intracellular trafficking of BDNF-containing vesicles is altered, resulting in a decreased release of BDNF and subsequent neuronal toxicity.

We therefore analyzed whether HSJ1b protein regulates BDNF release in neuronal cells. To make sure that any effect on BDNF release was not due to a defect in BDNF transcription, we ectopically expressed BDNF under the control of the CMV promoter. In addition, the levels of BDNF in the supernatant were normalized to BDNF levels in cell lysates. Mouse neuronal cells were lipofected with vectors expressing BDNF, HSJ1b or HSJ1a, or the corresponding empty vector, and the amount of BDNF in the supernatant was assessed by ELISA (Figure 3A). Strikingly, HSJ1b significantly increased the amount of BDNF released, whereas HSJ1a had no effect. These results suggest that HSJ1b promotes neuronal survival by enhancing BDNF release in neuronal cells.

We next examined whether the effect of HSJ1b could be recapitulated by cystamine. Treating cells with cystamine for varying lengths of time led to increased BDNF release (Figure 3B). We also analyzed the amount of BDNF in cell lysates and supernatants by standard Western blotting (Figure 3C). While the levels of BDNF were similar in cell lysates, 24 and 48 hours of cystamine treatment resulted in increased BDNF release. These findings show that HSJ1b and cystamine increase BDNF release in neuronal cells. This effect was specific to HSJ1b, as HSJ1a did not modify BDNF release under the same conditions.

To determine whether the effect of cystamine on BDNF secretion requires HSJ1b, we used an RNA interference approach. We transfected neuronal cells with pSUPER-RNAi-HSJ1, which targets both HSJ1a and HSJ1b, as we could not identify RNAi discriminating between the 2 isoforms (data not shown). We used RT-PCR to demonstrate that pSUPER-RNAi-HSJ1 reduced HSJ1 mRNA levels (Figure 3D). Strikingly, the ability of cystamine to increase BDNF release was lost in pSUPER-RNAi-HSJ1-transfected cells (Figure $3 \mathrm{E})$ showing that the effect of cystamine on BDNF release requires HSJ1 proteins. As HSJ1a is almost undetectable in brain and has no effect on BDNF secretion (Figure 3A), the results of this experiment strongly support the notion that at least part of the effect of cystamine on BDNF release involves HSJ1b.

$H S J 1 b$ stimulates the secretory pathway. Clathrin is the main component of the protein coats that decorate the cytoplasmic face of vesicles budding from the plasma membrane, the trans-Golgi network (TGN), and endosomes (37). The clathrin coat is, therefore, an important target for regulating secretion and endocytosis. To understand how HSJ1b and cystamine might act through clathrin to promote BDNF release, we first analyzed the distribution of HSJ1b, BDNF, and clathrin by performing subcellular fractionation of neuronal cells expressing HSJ1b and BDNF-GFP constructs (Figure 4A). We found that these 3 proteins are enriched in the $\mathrm{P} 3$ fraction corresponding to small vesicles, confirming the distribution of BDNF and cofractionating with huntingtin (27). We also purified CCVs and found they were enriched in HSJ1b and 
A

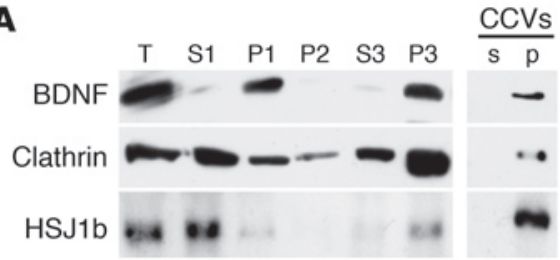

D

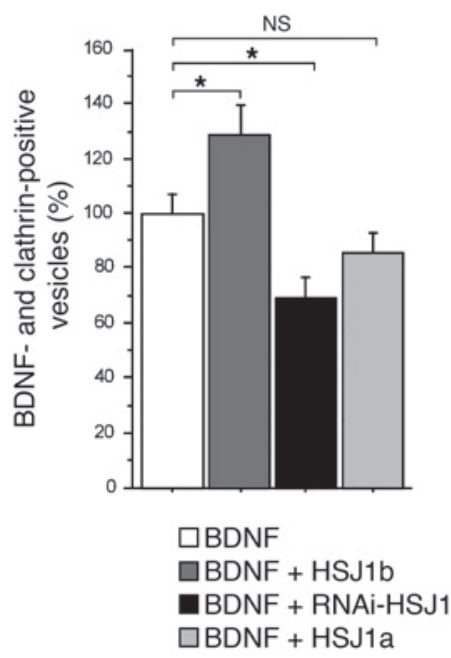

B

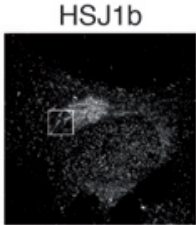

C

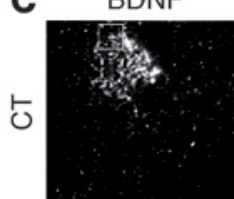

$\stackrel{5}{5}$
$\frac{1}{1}$
+
+
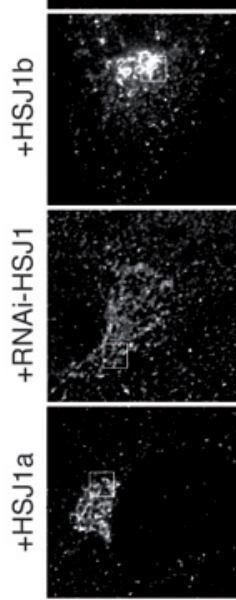

Golgi

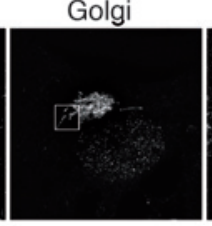

Clathrin
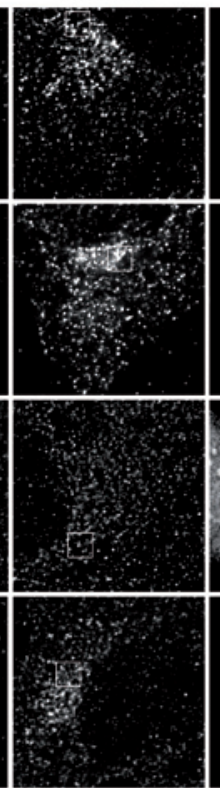

ER

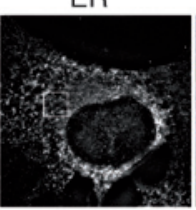

DAPI
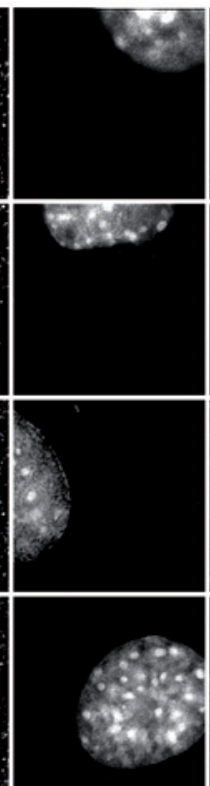

Merge

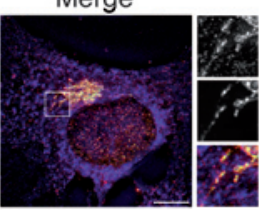

Merge

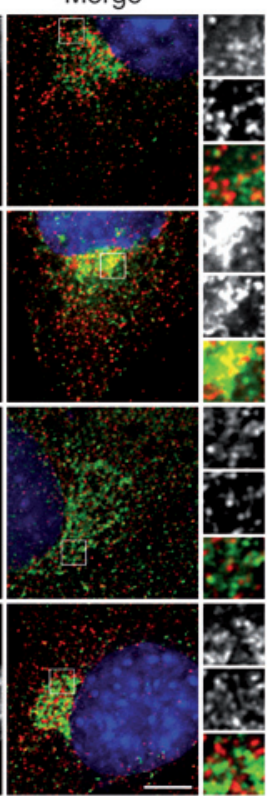

E

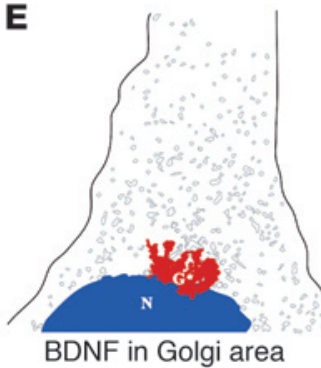

BDNF in Golgi area

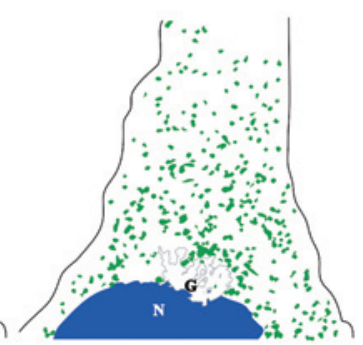

Cytoplasmic BDNF vesicles
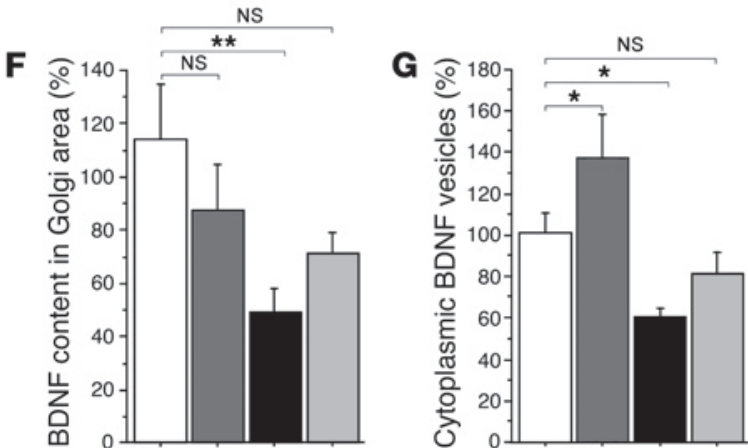

Figure 4

HSJ1b increases BDNF processing from the Golgi to the cytoplasm. (A) BDNF, clathrin, and HSJ1b were present in the same cellular compartments: small vesicle fraction (P3) and CCV fraction (p). (B) HSJ1b partially colocalized with the Golgi apparatus. (C) HSJ1b enhanced colocalization between BDNF and clathrin in the Golgi region, whereas RNAi-HSJ1 disrupted it. Scale bars: $10 \mu \mathrm{m}$. (D) Quantification (ANOVA, $F_{3,64}=7.71$; $P=0.0002$ ) revealed that expression of HSJ1b significantly increased the amount of BDNF vesicles that were clathrin positive compared with control cells (post-hoc Fisher's test, $P=0.032$ ), while pSUPER-RNAi-HSJ1 significantly decreased it (post-hoc Fisher's test, $P=0.019$ ). Overexpression of HSJ1a had no effect (NS). (E) Scheme showing the measurement of BDNF in the Golgi area (left) and in the cytoplasm (sorted vesicles, right). $(F)$ Quantification (ANOVA, $F_{3,67}=2,92 ; P=0.04$ ) revealed that lowering HSJ1b by interference significantly decreased BDNF content in the Golgi area (post-hoc Fisher's test, $P=0.005$ ). There was no difference between cells expressing HSJ1b or HSJ1a compared with control conditions (NS). (G) Quantification (ANOVA, $F_{3,47}=5,84 ; P=0.0018$ ) revealed that HSJ1b increased the amount of BDNF vesicles in the cytoplasm in comparison to control cells (post-hoc Fisher's test, $P=0.048$ ), whereas pSUPER-RNAi-HSJ1 decreased it (post-hoc Fisher's test, $P=0.038$ ). The difference was not significant in HSJ1a-overexpressing cells (NS). ${ }^{\star} P<0.05,{ }^{\star} P<0.01$.

BDNF. In addition, we found HSJ1b colocalizing with GMAP-210, a marker of the cis-Golgi (Figure 4B) (38) and with BDNF (Figure $5 \mathrm{E})$. These results are consistent with a role for $\mathrm{HSJ} 1 \mathrm{~b}$ in the regulation of BDNF processing in the secretory pathway.

Since HSJ1b is known to regulate clathrin coating in vitro, we analyzed by immunofluorescence microscopy the subcellular loca- tion of BDNF relative to the location of endogenous clathrin (Figure $4 \mathrm{C}$, upper panels). BDNF-containing vesicles and clathrin were both found predominantly in the Golgi region (as identified by immunostaining for GM130, another cis-Golgi marker; Figure 4C and data not shown). Increasing the levels of HSJ1b enhanced the colocalization of BDNF and clathrin, whereas reducing HSJ1b pro- 
A
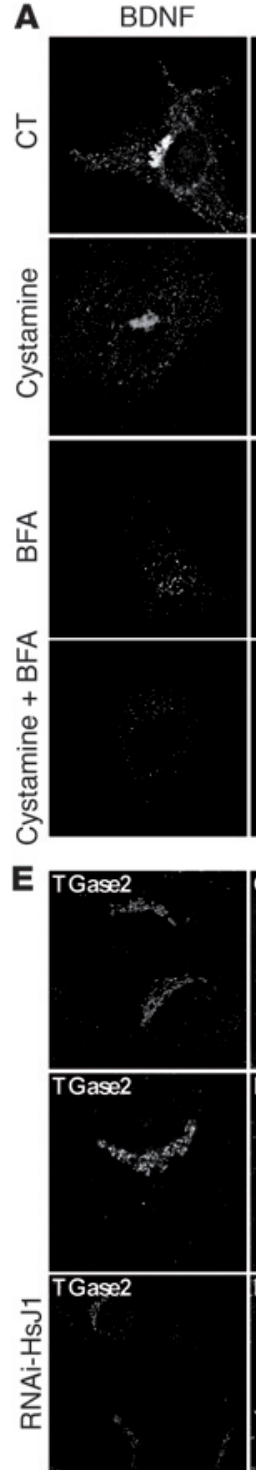

Golgi
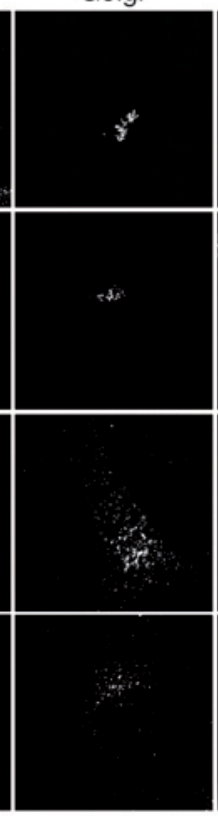

Golgi

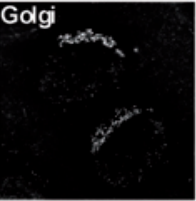

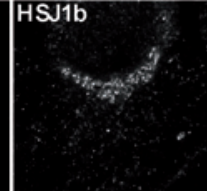

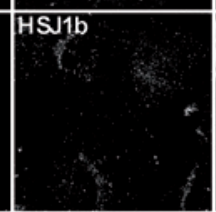

DAPI
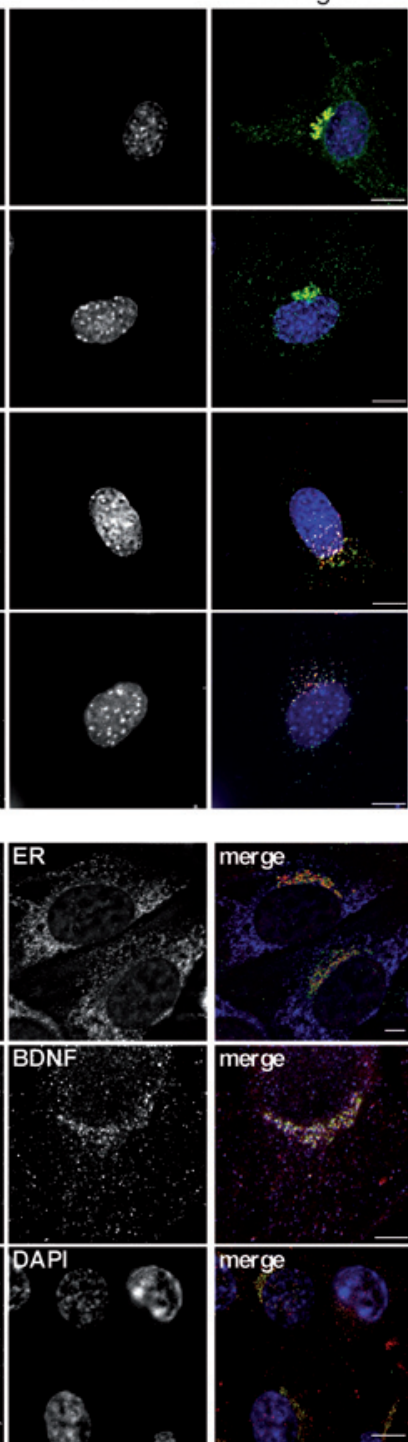

Merge
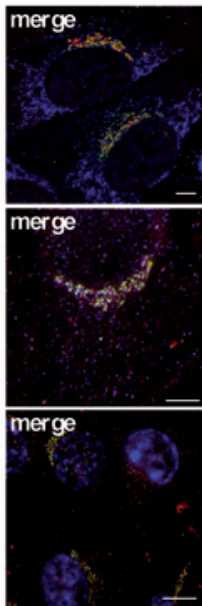

B
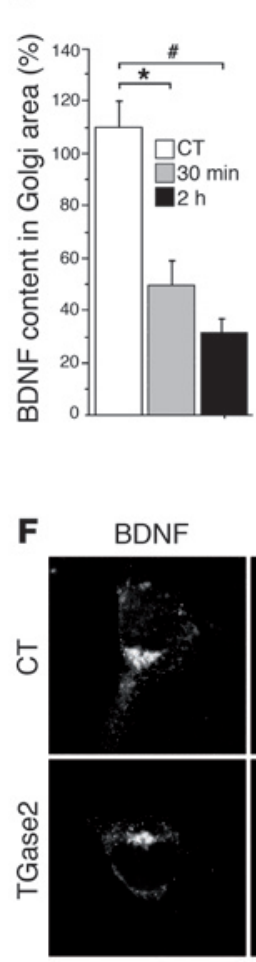

G

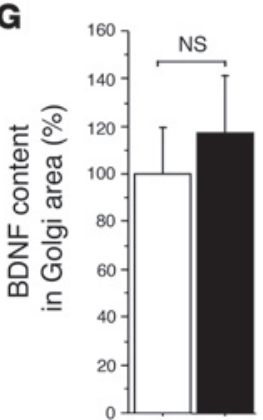

C

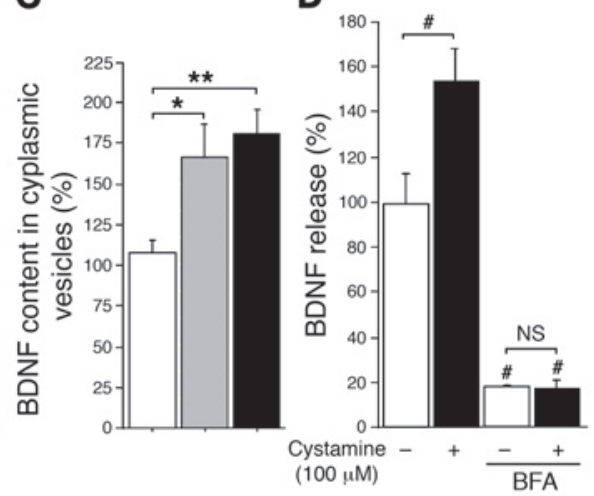

Golgi
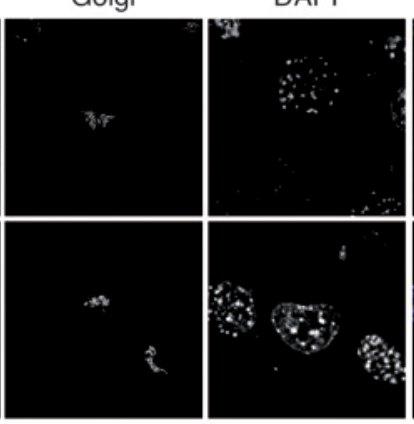

Merge

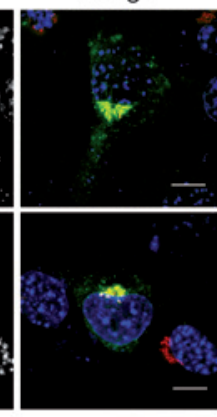

H

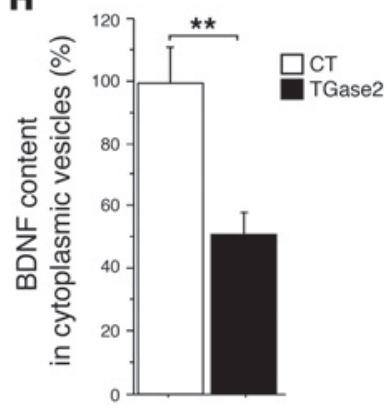

Figure 5

Cystamine and TGase 2 regulate BDNF secretion. (A) Cystamine treatment (100 $\mu \mathrm{M}, 30$ minutes) of BDNF-GFP-transfected cells decreased colocalization between BDNF and GM130. BFA dispersed BDNF vesicles and GM130 with or without cystamine. (B) Quantification (ANOVA, $\left.F_{2,46}=7.10 ; P=0.0021\right)$ revealed that cystamine significantly decreased BDNF in the Golgi area compared with control cells at 30 minutes (post-hoc Fisher's test, $P=0.011$ ) and 2 hours (post-hoc Fisher's test, $P=0.0007$ ). (C) Data (ANOVA, $\left.F_{2,46}=4.83 ; P=0.0125\right)$ revealed that cystamine treatment significantly increased BDNF content in cytoplasmic vesicles compared with control cells at 30 minutes (post-hoc Fisher's test, $P=0.021$ ) and 2 hours (post-hoc Fisher's test; $P=0.0049$ ). (D) Data (ANOVA, $F_{3,32}=45.6 ; P<0.0001$ ) revealed that BFA significantly reduced BDNF release in control and cystamine-treated cells (post-hoc Fisher's test, $P<0.0001$ ). Cystamine had an effect on control (post-hoc Fisher's test, $P<0.006$ ) but not on BFA-treated cells (NS). (E) TGase 2 colocalization with HSJ1b at the Golgi (GMAP-210) was disrupted in pSUPER-RNAi-HSJ1-transfected cells. (F) TGase overexpression induced a decrease in cytoplasmic BDNF-containing vesicles. Scale bars: $10 \mu \mathrm{m}$. (G) TGase 2 did not modify BDNF content in the Golgi area compared with control cells (Student's $t$ test, $t_{[13]}=0.4 ; \mathrm{NS}$ ). (H) TGase 2 induced a statistically significant decrease in cytoplasmic BDNF vesicles compared with control cells (Student's $t$ test, $\left.t_{[13]}=3.4 ; P=0.0051\right)$. ${ }^{\star} P<0.05,{ }^{*} P<0.01$, and ${ }^{\#} P<0.001$.

tein levels by RNA interference dramatically decreased it (Figure 4C, middle panels). Interestingly, decreasing HSJ1b protein levels also had an important impact on the intracellular distribution of clathrin, which appeared randomly distributed in the cytoplasm. HSJ1a had little or no effect on the colocalization of BDNF and clathrin. By quantifying the overlap between BDNF and clathrin, we found these effects to be statistically significant (Figure 4D).
This increase in the clathrin coating of BDNF-containing vesicles in response to overexpression of HSJ1b is consistent with the fact that HSJ1b inhibits hsc70-catalyzed clathrin uncoating (35).

To understand how an increase in the clathrin coating of BDNF vesicles leads to an increase in the release of BDNF from neuronal cells, we measured the sorting of BDNF from the Golgi/TGN region into the cytoplasm by image analysis. We quantified the 

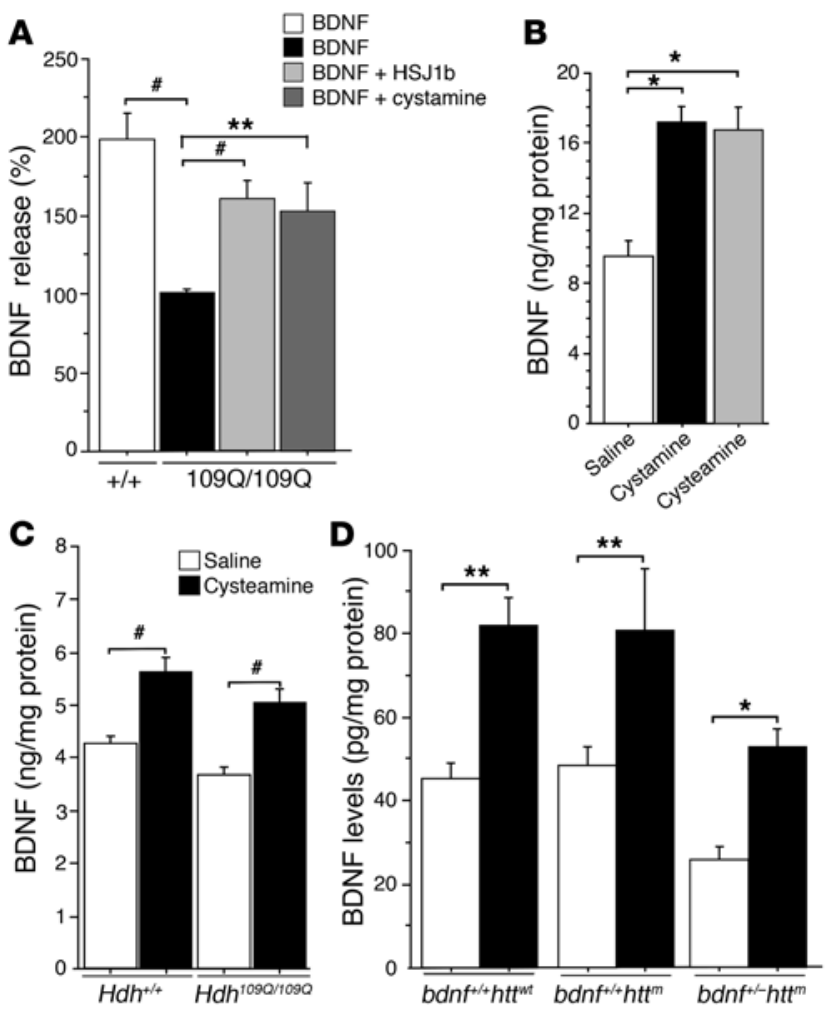

intensity of the BDNF signal in the Golgi region (Figure 4E, left) and the number of cytoplasmic BDNF-containing vesicles of 67 $\mathrm{nm}-3.35 \mu \mathrm{m}$ diameter (1-50 pixels) that correspond to vesicles budding from the Golgi/TGN region (Figure 4E, right). Reducing HSJ1 by RNA interference induced a statistically significant decrease in the BDNF content in the Golgi region (Figure 4F) and in the number of budding vesicles (Figure 4G). By contrast, HSJ1b overexpression increased the number of BDNF-containing vesicles in the cytoplasm. These findings indicate that HSJ1b is required for the formation of BDNF-containing vesicles.

Cystamine promotes BDNF secretion by an HSJ1b-dependent mechanism involving TGase inhibition. We have demonstrated that cystamine and HSJ1b increase BDNF release from neuronal cells and that the cystamine-induced BDNF release requires HSJ1b. We next treated neuronal cells with cystamine and analyzed the sorting of BDNF vesicles from the Golgi (Figure 5A). Analysis of 15-20 cells revealed that cystamine induced an extensive depletion of $\mathrm{BDNF}$ from the Golgi apparatus (Figure 5B) and increased the BDNF content of the vesicles (Figure 5C). These results indicate that cystamine promotes BDNF secretion by increasing the load of the neurotrophic factor in vesicles.

To verify that cystamine affects the secretion from the Golgi, we used brefeldin A (BFA), a fungal metabolite that is a potent inhibitor of Golgi vesicle fusion (39). BFA treatment led to dispersion of BDNF vesicles and, as expected, of the Golgi marker GM130 and was unaffected by cystamine treatment (Figure 5A, lower panels). We next analyzed BDNF release by ELISA in these conditions (Figure 5D). Strikingly, the ability of cystamine to increase BDNF release was lost in BFA-treated cells, demonstrating that the effect of cystamine on BDNF release requires secretion from the Golgi.

Because cystamine is well known to inhibit TGase in vitro, we tested the possibility that TGase might play a role in BDNF secre-

\section{Figure 6}

HSJ1b, cystamine, and cysteamine regulate BDNF levels in HD. (A) BDNF release was decreased (ANOVA, $F_{3,33}=17.45 ; P<0.0001$ ) in $109 \mathrm{Q} / 109 \mathrm{Q}$ cells compared with control cells (post-hoc Fisher's test, $P<0.0001$ ). This decrease was rescued when cells were transfected with HSJ1b (post-hoc Fisher's test, $P<0.0001$ ) or treated with $100 \mu \mathrm{M}$ cystamine for 30 minutes (post-hoc Fisher's test, $P=0.001$ ). (B) Data (ANOVA, $F_{2,29}=3.63 ; P=0.0392$ ) revealed a statistically significant increase in the amount of BDNF in the brains of mice treated with cystamine (post-hoc Fisher's test, $P=0.015$ ) or with cysteamine (post-hoc Fisher's test, $P=0.04$ ) compared with controls. (C) Data (ANOVA, $F_{3,49}=11.66$; $P<0.0001$ ) revealed a statistically significant increase in the amount of BDNF in the brains of mice treated with cysteamine (post-hoc Fisher's test, $P<0.001$ ). (D) Cysteamine increased BDNF levels in the striatum of the different genotypes (ANOVA, $F_{5,14}=9.20 ; P<0.0004$ ): wild-type animals (post-hoc Fisher's test, $P=0.002$ ); $b d n f^{+/+} h t^{m}$ mice (R6/1mice; posthoc Fisher's test, $P=0.009$ ); and $b d n f^{+/-} h t t^{m}$ mice (post-hoc Fisher's test, $P=0.024)$. ${ }^{\star} P<0.05,{ }^{* \star} P<0.01$, and ${ }^{\#} P<0.001$.

tion. Several isoenzymes of TGase are found in the brain, of which TGase 2 is the most abundant. We found that TGase 2 partially colocalized with GMAP-210, with HSJ1b, and with BDNF (Figure $5 \mathrm{E}$, upper and middle panels). We next analyzed whether TGase 2 localization depends on HSJ1b. Interestingly, reducing HSJ1b levels by RNA interference modified TGase 2 localization at the Golgi (Figure 5E, lower panels) and disrupted the Golgi (data not shown). We also assessed the consequences of increased TGase 2 activity on BDNF secretion. Cells were transfected with TGase 2 and treated with glutamate ( $5 \mu \mathrm{M}, 30$ minutes) to increase intracellular calcium concentration and subsequently activate the enzyme (40). In these conditions, BDNF remained in the Golgi area, whereas the number of BDNF-containing vesicles in the cytoplasm was reduced (Figure 5, F-H). These results demonstrate that TGase 2 inhibits BDNF processing. This observation is consistent with a report showing that TGase inhibitors stimulate catecholamine release from rat brain synaptosomes (41).

We have shown that cystamine induces BDNF release by increasing its processing from the Golgi/TGN region. We propose that cystamine acts at 2 levels: first, it stimulates the transcription of HSJ1b that is required for the sorting of BDNF-containing vesicles and second, it also increases BDNF processing by inhibiting TGase activity.

$H S J 1 b$ and cystamine rescue BDNF release defects induced by polyQbuntingtin in cells and in HD mice. We determined the effect of HSJ $1 \mathrm{~b}$ and cystamine on BDNF release in the pathological situation. We used mouse neuronal cells derived from knock-in mice in which a 109-CAG expansion, encoding 109 glutamine residues, was inserted into the endogenous mouse huntingtin gene (109Q/109Q) (28). This cell line closely resembles the situation in HD patients as, in these cells, polyQ-huntingtin is expressed at endogenous levels. The level of BDNF released from these cells into the supernatant was significantly lower in 109Q/109Q cells compared with wild- 
A
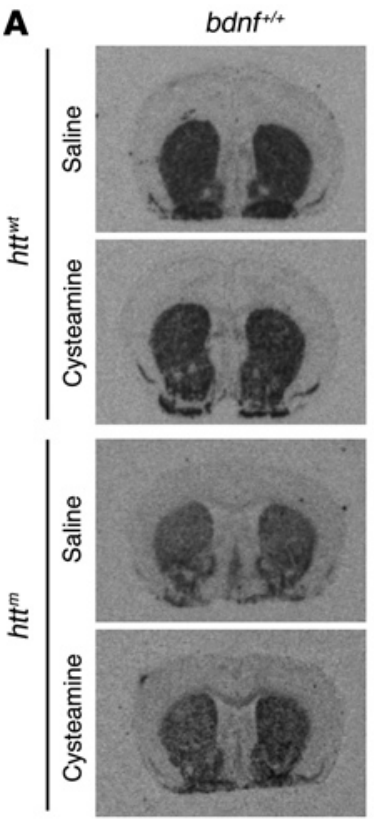

B
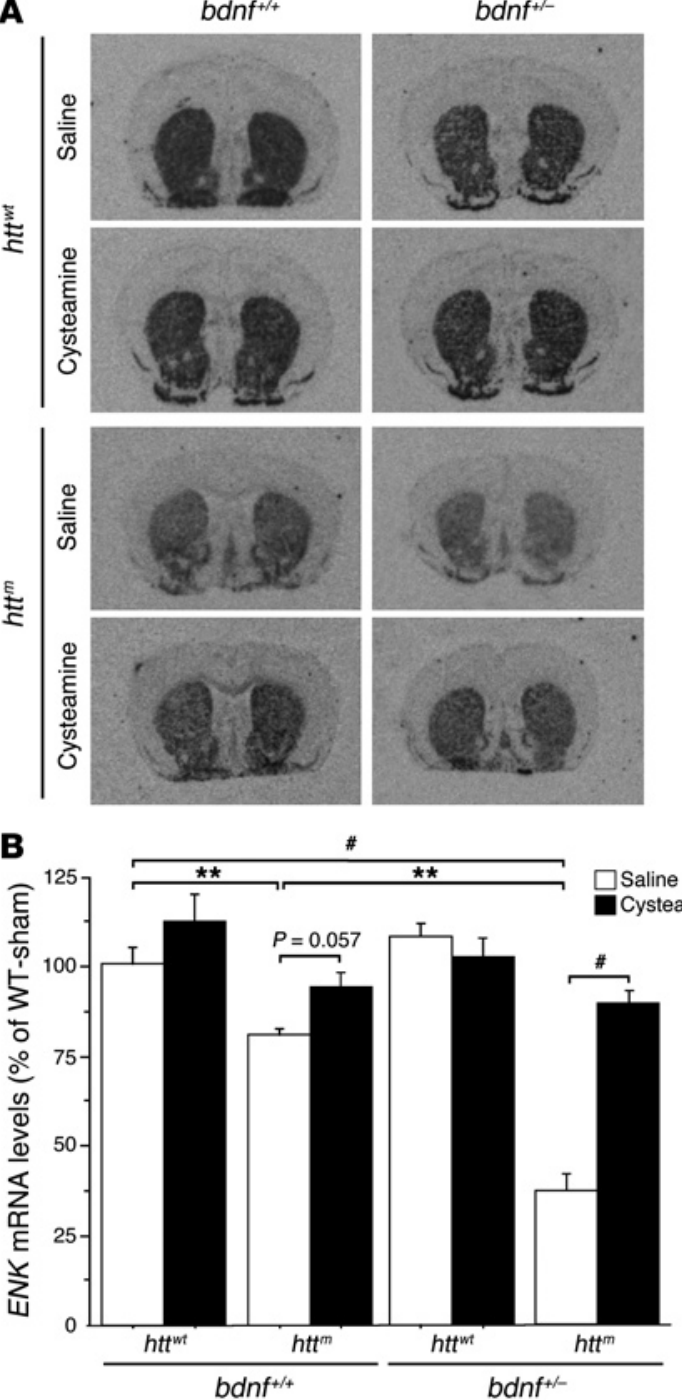

C

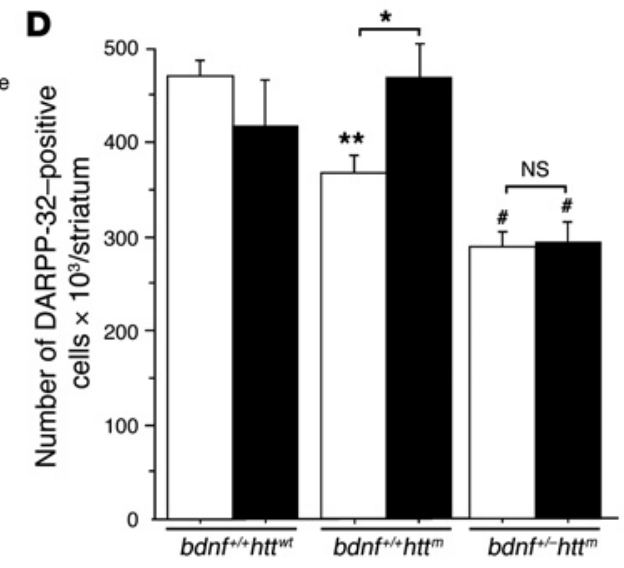

Figure 7

Cysteamine is neuroprotective in HD mice through a BDNF-dependent mechanism. (A) In situ hybridization revealed that ENK mRNA were reduced in R6/1 (bdnft++htt $)$ and dramatically reduced in bdnf+l-httm mice. At early stages, cysteamine treatment increased the levels of ENK mRNA in bdnf+/+httm and in bdnf+/-httm mice. (B) Quantification of ENK mRNA levels. WT-sham, wild-type saline-treated mice. (C) Cysteamine treatment of wild-type animals did not modify DARPP-32 immunostaining. In R6/1 animals, there was a decrease in the number of DARPP-32positive cells that was recovered by cysteamine treatment. The loss of DARPP-32-positive neurons could not be prevented by cysteamine treatment in bdnf+/-htt ${ }^{m}$ mice. Scale bar: $50 \mu \mathrm{m}$. (D) Quantitative analysis of the changes in DARPP-32 immunostaining. ${ }^{*} P<0.05,{ }^{* \star} P<0.01$, and ${ }^{\#} P<0.001$; see statistical analysis in Methods.

type neuronal cells (Figure 6A). Interestingly, ectopic expression of HSJ1b or treatment with cystamine increased BDNF release from 109Q/109Q cells. Thus, the defect in BDNF release in a cellular model of HD can be rescued by HSJ1b or cystamine.

We next investigated whether cystamine regulates BDNF levels in the brain by injecting mice daily with cystamine. Mice were sacrificed after 7 days of treatment (30 minutes after the last injection), and the BDNF levels in brain extracts were measured. Treatment with cystamine resulted in an increase in brain BDNF level (Figure $6 \mathrm{~B}$ ). We also assessed in vivo the effect of the reduced form of cystamine, cysteamine. Unlike cystamine, cysteamine is an FDAapproved drug that is used to treat a rare childhood disorder called cystinosis (42), and tolerability of cysteamine was determined in HD patients $(43,44)$. Therefore, cysteamine might be used immediately after appropriate phase II clinical studies for use in HD patients. We analyzed the ability of cysteamine in comparison to cystamine to increase BDNF levels in the brain of treated mice and observed that cysteamine recapitulated the positive effect of cystamine on BDNF secretion in animals (Figure 6B). Having shown that cystamine and cysteamine are effective in wild-type mice, we tested the ability of cysteamine to regulate BDNF release in knock-in mice carrying a 109Q repeat in the $H d h$ locus that are homozygous for the mutation (45). We observed a small but not significant reduction $(P=0.096)$ in the brain levels of BDNF in $H d b^{1090 / 109 Q}$ mice compared with their wild-type littermates (Figure 6C). Interestingly, cysteamine induced a significant increase in BDNF levels in HD knock-in mice. 
A
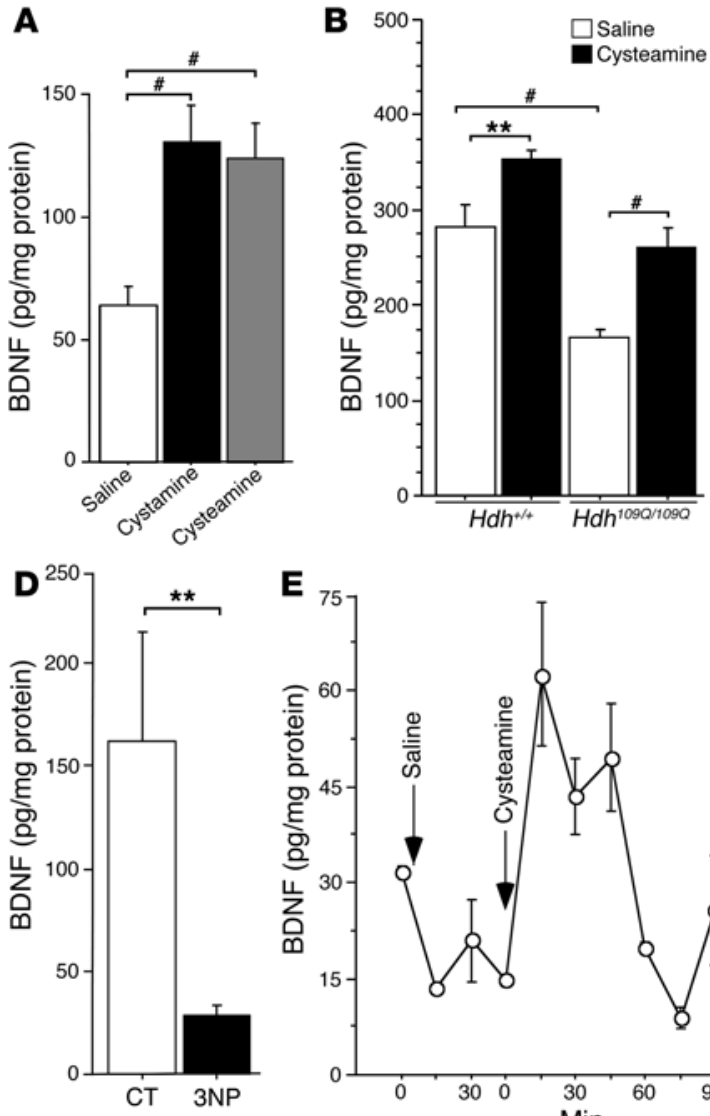

$\mathbf{E}$

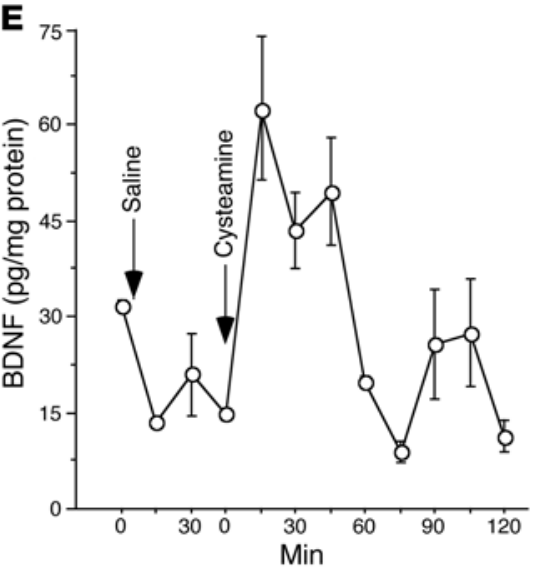

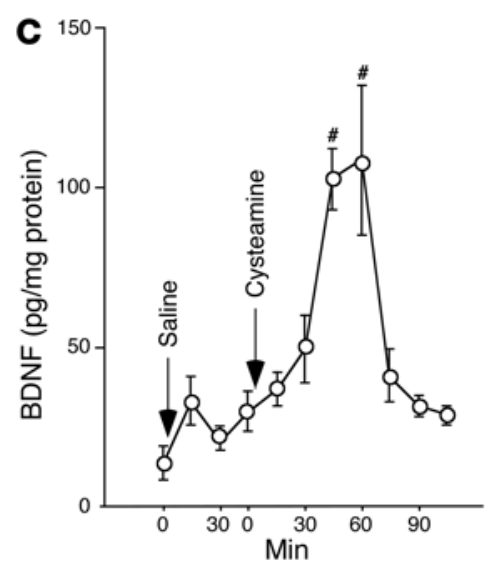

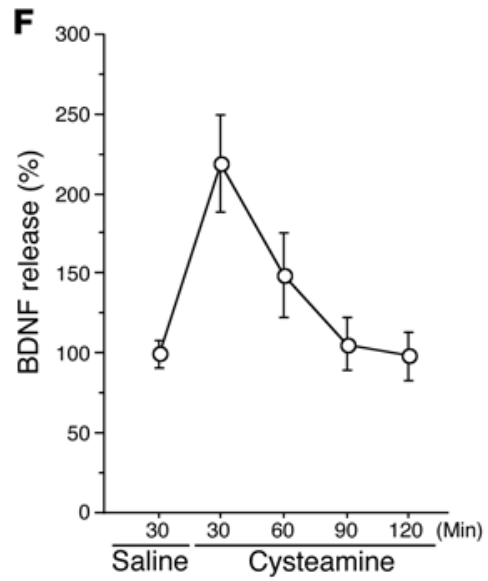

Figure 8

Cysteamine increases blood levels of BDNF in rodents and in a primate HD model. (A) Data (ANOVA, $F_{2,27}=17.13 ; P<0.0001$ ) revealed that the amount of BDNF in serum of mice treated with cystamine (posthoc Fisher's test, $P<0.0001$ ) or with cysteamine (post-hoc Fisher's test, $P<0.0001$ ) was significantly increased. (B) The blood level of BDNF (ANOVA, $F_{3,50}=17.22 ; P<0.0001$ ) was reduced in $H d h^{109 Q / 109 Q}$ mice compared with wild-type littermates (post-hoc Fisher's test, $P=0.0001$ ) and rescued by cysteamine. The amount of BDNF in the serum of wild-type mice (post-hoc Fisher's test, $P=0.0099$ ) and $\mathrm{Hdh}^{109 Q / 109 Q}$ mice (post-hoc Fisher's test, $P=0.0010$ ) was increased upon cysteamine treatment. (C) There was an increase of BDNF in serum of 4 cysteamine-treated rats (ANOVA, $F_{10,64}=9.29 ; P<0.0001$ ) after 45 minutes (post-hoc Fisher's test, $P<0.0001$ ) and 60 minutes (post-hoc Fisher's test, $P<0.0001$ ). (D) BDNF levels were reduced in the blood of 3NP-treated monkeys (Student's $t$ test, $t_{[27]}=3.05 ; P=0.005$ ). ${ }^{* \star} P<0.01$ and $\# P<0.001$. Data are from 4 controls and three 3NP-treated monkeys. (E and $\mathbf{F}$ ) Blood BDNF levels were increased in HD monkeys injected with cysteamine. (E) Representative results for 1 monkey are shown. (F) Graph represents the results for the 2 injected monkeys. Error bars represent SEM.

and late (26 weeks) stages of the disease. At 16 weeks, R6/1 mice are still presymptomatic, while double mutant $\left(b d n f^{+/-} b t t^{m}\right)$ mice already have a motor behavioral impairment. We analyzed the mice for enkephalin, a specific marker for striatopallidal neurons that are affected early in HD, and substance $\mathrm{P}$, a marker for striatonigral neurons. At this early stage, enkephalin mRNA levels were not highly affected in R6/1 mice, whereas a much greater reduction was observed in $b d n f^{+/-} h t t^{m}$ mice (Figure 7, A and B) (26). Administration of cysteamine in double mutant mice increased the expression of enkephalin above the levels in sham-R6/1 and sham$b d n f^{+/-} b t t^{m}$ mice. Cysteamine treatment did not modify the number of substance P-positive neurons in $\mathrm{R} 6 / 1$ mice or in double mutant mice above that in control mice (Supplemental Figure 2). These data show that cysteamine is neuroprotective in R6/1 mice at early stages.

We next determined changes in dopamine and cAMP-regulated phosphoprotein of a molecular mass of $32 \mathrm{kDa}$ (DARPP-32), a specific marker for striatal projection neurons that is affected at the age of 26 weeks, but not at 16 weeks, in R6/1 mice. As expected, at 26 weeks, a decrease in DARPP-32positive cells was observed in R6/1 compared with wild-type mice (Figure 7C). Interestingly, whereas cysteamine treatment

Cysteamine is neuroprotective in R6/1 mice by increasing BDNF levels. Strong evidence indicates that cystamine is neuroprotective in HD mouse models $(10,11,14,15)$. We aimed to assess whether cysteamine is neuroprotective in vivo and whether this depends on BDNF. For this purpose, we used a mouse model of HD, R6/1 mice, which show a much faster progression of disease phenotype compared with $H d b^{1090 / 109 Q}$ mice $(46,47)$ and a double mutant line previously generated by crossing R6/1 mice with $b d n f$ heterozygous mice (26). We first investigated whether cysteamine regulates BDNF levels in the striatum by injecting wild-type, R6/1 $\left(b d n f^{+/+} b t t^{m}\right)$, and double mutant mice ( $\left.b d n f^{+/-} b t t^{m}\right)$ daily for 7 days (Figure 6D). Although less efficiently in $b d n f^{+/-} b t t^{m}$ mice, this treatment induced an increase in striatal BDNF levels in the 3 mouse genotypes.

We next analyzed the effect of cysteamine treatment in different neuronal populations in these HD models at early (16 weeks) prevented the reduction in the number of DARPP-32-positive cells in $\mathrm{R} 6 / 1$ mice, this treatment had no effect in $\mathrm{R} 6 / 1$ mice with disruption of $b d n f\left(b d n f^{+/-} b t t^{m}\right.$ mice). We quantified these changes and found them to be statistically significant (Figure 7D).

Taken together, the results of experiments using enkephalin and DARPP-32 markers indicate that cysteamine is also neuroprotective in HD mice. This effect is similar to that of exogenous BDNF administration (26). Furthermore, these data demonstrate that this neuroprotective effect is dependent on the levels of endogenous BDNF.

Cystamine and cysteamine increase blood levels of BDNF in rodent and primate models of $H D$. We explored whether the positive effect of cystamine and cysteamine on BDNF secretion can also be seen in peripheral tissues. As with brain extracts, we observed a similar increase in BDNF levels in the blood of mice treated with cystamine or with cysteamine (Figure 8A). We next asked whether such chang- 
es can be seen in $H d h^{1090 / 109 Q}$ mice (Figure 8B). The blood of mice for which brain BDNF levels were measured (Figure 6C) was analyzed. Interestingly, a much greater reduction in the level of BDNF was detected in the serum of $H d h^{1090 / 109 Q}$ mice compared with wild-type mice. As for brain levels, cysteamine also increased BDNF levels in $H d h^{1090 / 109 Q}$ mice. We next characterized the pharmacokinetics of cysteamine's effect on BDNF levels in the blood of rats injected with cysteamine (Figure 8C). As in mice, blood BDNF levels also increased in response to cysteamine in rats. The maximum increase was reached 45-60 minutes after cysteamine administration, and BDNF levels returned to basal level after 90 minutes.

To further explore the effect of cysteamine in a situation that is close to human pathology, we chose a nonhuman primate model of HD produced by chronic systemic administration of the mitochondrial complex II inhibitor 3-nitropropionic acid (3NP). Chronic $3 \mathrm{NP}$ treatment produces striatal dysfunction and neurodegeneration in primates, leading to abnormal movements (including choreiform movements and dystonia) and frontal-type cognitive deficits that are highly reminiscent of $\operatorname{HD}(48,49)$. We first asked whether BDNF levels were altered in the blood of 3 NP-treated primates. We analyzed blood samples from 3NP-treated long-tailed macaques and observed a statistically significant decrease in peripheral BDNF when compared to control animals (Figure 8D).

Having shown that peripheral BDNF is decreased, we tested whether systemic injection of cysteamine would enhance BDNF levels in the primate model of HD. In two 3NP-treated macaques, we found that acute injection of cysteamine $(100 \mathrm{mg} / \mathrm{kg})$ rapidly induced an increase in BDNF blood levels (Figure 8, E and F). As in rats, the blood levels of BDNF peaked around 30 minutes after cysteamine injection and progressively returned to normal within 60 minutes, consistent with a transient but massive increase in BDNF release into the plasma compartment. This demonstrates that in a primate model of HD, BDNF peripheral levels are decreased and can be increased by administration of cysteamine.

\section{Discussion}

Cystamine is one of the very few candidate drugs being considered for the treatment of HD. Here we have investigated the molecular mechanisms of action of cystamine, and our study has revealed that an FDA-approved reduced form of cystamine produces very similar biological effects in vitro and in vivo. We demonstrate that part of the neuroprotective effect of cystamine is due to its promotion of secretion of the neuronal survival factor BDNF. Cystamine has 2 quite distinct actions in this regard. First, it increases the steadystate levels of the Hsp HSJ1b mRNA, which stimulates the secretory pathway through its action on CCV formation, and, second, it inhibits TGase, which has a negative effect on BDNF sorting.

HSJ1b belongs to the large family of DnaJ-like proteins that contain the typical Hsp40 chaperones HDJ1/Hsp40 and HDJ2/HSDJ. In most cases, these chaperones have been reported to reduce polyQinduced aggregation and toxicity in various models (for reviews, see refs. 20-22). We found that HSJ1b functions in a qualitatively different way inasmuch as it appears not to prevent aggregation or the formation of NIIs. However, we found that HSJ1b is relevant to HD, as it strongly inhibits polyQ-huntingtin-induced neuronal death in vitro and rescues neuronal dysfunction in a nematode model of HD. Although we found that in mammalian cells, HSJ1b stimulates BDNF secretion, the exact mechanism by which HSJ1b operates in nematodes remains to be established. Indeed, BDNF is absent in nematodes. HSJ1b could, however, enhance the secretion of other specific C. elegans factors. In C. elegans, trophic factors other than $\mathrm{BDNF}$ exist. In particular, the mesencephalic, astrocyte-derived neurotrophic factor, MANF, is well conserved from C. elegans to humans and could be the target of HSJ1b (50). Furthermore, BDNF second messengers are conserved. Therefore, it is possible that HSJ1b acts through a pathway involving these molecules.

In mammalian cells the neuroprotective properties of HSJ1b are linked to its ability to enhance neurotrophic support. HSJ1b positively regulates the sorting of BDNF-containing vesicles from the Golgi/TGN, leading to an increase in BDNF release. These findings are in agreement with the function of HSJ1b in the inhibition of the uncoating of CCVs (35). Most of the vesicles budding from the Golgi/TGN region are CCVs, and assembly of the clathrin coat on the forming bud is an important step (37). HSJ1b could therefore promote the budding of BDNF-containing vesicles by stabilizing this assembly step. Consistent with this idea, we observed an increased percentage of BDNF-containing vesicles that were clathrin positive when HSJ1b expression was increased, whereas reducing HSJ1b levels decreased it. Interestingly, we found significantly less HSJ1b in postmortem brain extracts from HD patients than from control brains, suggesting a potential alteration in the processing of BDNF at the Golgi/TGN during HD pathogenesis. A defect in clathrin coating processes in HD is supported by the fact that huntingtin interacts with HIP1 and HIP12/HIP1R, 2 proteins that are components of clathrin coats and that regulate clathrin assembly by directly interacting with clathrin $(51,52)$. HSJ $1 \mathrm{~b}$ is thought to inhibit uncoating by interfering with the interactions of Hsc70 with specialized uncoating DnaJ-like proteins such as auxilin (35). Whether it also regulates the activity of huntingtin interactors such HIP1 remains to be determined.

The second consequence of cystamine treatment is promotion of the secretion of BDNF vesicles through a mechanism involving TGase. Our findings that TGase 2 colocalizes with BDNF at the Golgi and the observation that TGases regulate the secretion of BDNF is consistent with previous observations from the $1980 \mathrm{~s}$ indicating a role of TGases in inhibiting secretion and/or release of various hormones and neurotransmitters such as insulin, serotonin, and dopamine $(41,53,54)$. For instance, monodansylcadaverine (MDC), a potent TGase inhibitor, enhances dopamine release from rat brain synaptosomes in basal and in potassiumstimulated conditions (41). Moreover, reports that MDC blocks clathrin-mediated endocytosis $(55,56)$ accord with our finding that cystamine and TGases regulate the clathrin pathway.

We and others have reported a lack of BDNF support in HD that involves defects in BDNF synthesis (25) and transport (27). Our findings suggest that, in addition, a defect in BDNF sorting from the Golgi/TGN occurs in HD and that such BDNF processing is regulated by HSJ $1 \mathrm{~b}$ and TGases.

Cystamine was first described as a TGase inhibitor in vitro, and several studies are consistent with the possibility that cystamine is beneficial in HD by this mechanism $(10,11,14,15)$. However, whether cystamine is directly inhibiting TGase 2 in vivo remains to be clearly established. First, the reduced form of cystamine, cysteamine, could act as a competitive inhibitor of TGase 2 in vivo (57). Second, other metabolites of cystamine and cysteamine could mediate the neuroprotective effect, as they are rapidly metabolized and low to undetectable levels of cystamine and cysteamine are found in the brain of cystamine-treated mice (17). In addition, cystamine or its metabolites could act through a TGase-independent mechanism (16). Cystamine inhibits caspase 3 activity and 
increases glutathione levels in cells (18). In vivo, the beneficial effect of cystamine could involve the increase in L-cysteine, which has antioxidant properties $(17,19)$.

We now show that cystamine and cysteamine target HSJ1b and TGase to increase the release of BDNF, a trophic factor that is depleted in HD and that is crucial for the survival of striatal neurons in HD. Our data further emphasize that cystamine or its metabolites acts at multiple levels to protect against polyQ-huntingtin-induced toxicity and therefore add to the motivation for optimizing a therapy with cystamine or related compounds.

We demonstrate that cysteamine is as efficient as cystamine in increasing levels of BDNF in the brain. We also report that cysteamine is neuroprotective in HD mice by increasing levels of BDNF in brain. BDNF levels can also be measured in blood as a biomarker for pathological stages. We found that in HD knock-in mice and in a primate model of $\mathrm{HD}$, the levels of BDNF in serum were reduced compared with those in controls, whereas such decreases in brain BDNF could not be detected in HD knock-in mice and in R6/1 mice at 15 and 16 weeks of age. Interestingly, at these early stages, these mice do not show overt phenotypes $(45,46)$. This suggests that blood BDNF could be used to follow disease progression and validate the neuroprotective effects of drugs acting on BDNF levels.

We found cysteamine-induced release of BDNF in brain to be transient. This is consistent with the rapid clearance of cysteamine from the plasma of healthy individuals (58) and patients with nephropathic cystinosis (59) and suggests that, as for the treatment of nephropathic cystinosis, repeated doses of cysteamine at short intervals would be appropriate for the treatment of HD. Such limited and controlled release of BDNF is of particular interest for therapy, as an excessive stimulation of the BDNF/TrkB pathway leads to tumorigenesis in mice (60). Moreover, the efficacy of a repeated treatment is unlikely to diminish with time, as we found that the cysteamine-induced increase in brain and serum BDNF levels was still detected after 12 weeks continuous treatment (Supplemental Figure 3).

We propose the use of cysteamine as a therapeutic approach to treat HD. Indeed, the safety of cysteamine in humans is well documented, as cysteamine is used to treat cystinosis $(42,61)$. Moreover, the tolerated cysteamine dose has been evaluated in HD patients $(43,44)$. Finally, our findings indicate that the efficacy of cysteamine treatment in HD patients could be monitored by measuring serum levels of BDNF as a convenient biomarker.

\section{Methods}

Constructs. The vectors encoding BDNF, BDNF-GFP, Hsp40, and TGase 2 have been described previously (62-64). The 171-17Q-HA and the 17173Q-HA were obtained by inserting an HA tag into the XhoI-XbaI sites of the pcDNA-171-17Q and pcDNA-171-73Q, respectively (24). The oligonucleotides used were: 5'-TCGAGTACCCATACGATGTTCCAGATTACGCTTAAT- ${ }^{\prime}$ ' and 5'-CTAGATTAAGCGTAATCTGGAACATCGTATGGGTAC-3'. The BamHI inserts of pBPSTR-1-HSJ1a and of pBPSTR-1-HSJ1b contained the sequences encoding human HSJ1a and HSJ1b, respectively (29), and were subcloned into the pcDNA3 vector (Invitrogen Corp.).

The RNA sequence targeting mouse HSJ1 corresponds to the coding region 97-116 (GenBank accession number NM178055). The pSUPER-RNAi-HSJ1 construct was generated by inserting in the BbsI cloning site of pSuperhH1Neo (InvivoGen) the following annealed oligonucleotides: $5^{\prime}$-CAAAAAGACAAGAACCCGGATAATAGGTGGTATTATCCGGGTTCTTGT-3' and 5'-TCCCGACAAGAACCCGGATAATACCACCTATTATCCGGGTTCTTGTCTT-3'.
Real-time RT-PCR. Forty-eight hours after transfection, neuronal cells (wild-type; ref. 28) were treated with $100 \mu \mathrm{M}$ cystamine (Sigma-Aldrich) for 24 or 48 hours and lysed in TRIzol (Invitrogen Corp.). Total RNA was extracted, and samples were retrotranscribed using the First-Strand cDNA Synthesis Kit (Amersham Biosciences). cDNAs were then diluted 1:400 and submitted to RT-PCR (iQ SYBR Green Supermix; Bio-Rad) with the following HSJ1 oligonucleotides corresponding to the mouse coding region 1272-1387 (GenBank accession number NM178055): 5'-TCAGGCCССТTCTTTACCTT-3' and 5'-AAGGGTCTCCACTCCCAAAAG-3'. HPRT gene was used as an internal control and quantified with the following oligonucleotides: 5'-CACAGGACTAGAACACCTGC-3' and 5'-GCTGGTGAAAAGGACCTCT-3'.

Results were analyzed using the ICycler apparatus (Bio-Rad). Data are from 4 separate quantitative RT-PCR experiments performed in triplicate from 2 independent mRNA preparations.

Human tissues. Human tissue samples 1-5 were from the Harvard Brain Tissue Resource Center (HBTRC) and correspond to brain numbers 4741, $4744,4751,4797$, and 4740 as numbered by the HBTRC. Samples 1-3 were controls (age: $55.3 \pm 1.9$ years; postmortem delay: $23.8 \pm 1.9$ hours $[$ mean \pm SEM]). Samples 4 and 5 were from grade 3 and 4 HD brains, respectively (age: $63.5 \pm 18.5$ years; postmortem delay: $24.0 \pm 2.0$ hours). Human brain samples 6-27 were collected at the Salpêtrière. Samples 6-10 were putamen from controls (age: $72.6 \pm 8.9$ years; postmortem delay: $9.4 \pm 2.1$ hours). Samples $11-15$ were putamen from symptomatic HD patients with family history (age: $65.6 \pm 3.7$ years; postmortem delay: $26.5 \pm 7.4$ hours). Samples $16-22$ were caudate samples from controls (age: $79.4 \pm 1.7$ years; postmortem delay: $13.8 \pm 3.0$ hours). Samples $23-27$ were caudate samples obtained from symptomatic HD patients with family history (age: $65.0 \pm 4.0$ years; postmortem delay: $25.2 \pm 7.4$ hours).

Brain samples were homogenized in NP40 lysis buffer $(20 \mathrm{mM}$ Tris- $\mathrm{HCl}$ pH 7.5, 150 mM NaCl, 2 mM EGTA, $1 \%$ Nonidet P-40, 10 mM $\beta$-glycerophosphate, $5 \mathrm{mM} \mathrm{NaF}, 1 \mathrm{mM} \mathrm{NaPPi}, 2 \mathrm{mM}$ DTT, $1 \mathrm{mM}$ sodium vanadate, $100 \mu \mathrm{M}$ PMSF) and cleared by centrifugation at $6,000 \mathrm{~g}\left(15\right.$ minutes; $\left.4^{\circ} \mathrm{C}\right)$. Fifty micrograms of homogenates were subjected to Western blot analysis. Quantifications of Western blots were performed and expressed relative to actin levels. Human biopsy samples were procured in accordance with the guidelines as adopted and promulgated by the NIH. Permission to perform the experiments with the samples was granted by the Scientific Advisory Board of the Harvard Brain Tissue Resource Center at McLean Hospital (Belmont, Massachusetts, USA).

Cell culture, transfection, and immunofluorescence. Primary cultures of striatal neurons were prepared from E17 Sprague-Dawley rats and transfected at 4 days in vitro by a modified calcium phosphate technique (24). Mouse neuronal cells derived from wild-type huntingtin mouse (neuronal cells, + /+) and from $H d h^{109 Q / 109 Q}$ knock-in mouse (109Q/109Q) were cultured as previously described (28) and transfected with Lipofectamine 2000 (Invitrogen Corp.). Human 293 T cells were cultured in DMEM supplemented with $10 \%$ bovine calf serum. Transfected neuronal cells were grown on glass coverslips, fixed with $4 \%$ paraformaldehyde for 20 minutes, and immunostained as previously described (27). For experiments represented in Figures 4B and 5E, cells were fixed with methanol/acetone (vol/vol) at $-20^{\circ} \mathrm{C}$ for 10 minutes.

Measurement of neuronal survival and intranuclear inclusions. Four days after plating, primary cultures of striatal neurons were transfected with wild-type or polyQ-huntingtin and GFP to identify the transfected cells. To be certain that each neuron synthesizing GFP also expressed the huntingtin construct, transfections were performed using a derived phosphate calcium method with a high ratio of huntingtin DNA to GFP DNA (10:1) (65). Under these conditions more than $95 \%$ of the GFP-positive neurons also expressed the huntingtin construct (data not shown). GFP-positive neurons were scored using fluorescence microscopy in a blinded manner 16 hours and 36 hours 
after transfection. Cell death occurring within the GFP-positive cells was determined as the difference in the number of surviving neurons between the 2 time points and expressed as percentage of cell death. For intranuclear inclusions scoring, striatal neurons were transfected with vectors of interest and a plasmid encoding $\beta$-galactosidase (10:1). Neurons were fixed 5 days after transfection, immunostained, and analyzed for the presence of ubiquitin-positive intranuclear inclusions (anti- $\beta$-galactosidase, 1:300; 5 Prime-3 Prime Inc.; anti-ubiquitin, 1:100; Dako). Each graph represents 2-3 independent experiments performed in triplicate. Each bar in a given graph corresponds to the scoring of about 2,000 neurons in neuronal survival experiments and 500 neurons for inclusions scoring.

C. elegans assays. Nematode strains were handled following traditional methods (66). All strains were received from the Caenorhabditis Genetics Center, University of Minnesota. Construction of huntingtin transgenics, touch response assays, and visualization of neurons were carried out as described previously $(32,33)$. Transgenic strains expressing human HSJ1b were constructed by microinjection of lin-15(n765ts) animals with a mix

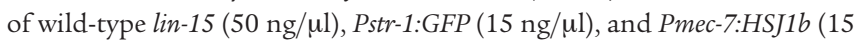
$\mathrm{ng} / \mu \mathrm{l})$. Independent stable lines were established, and 4 lines with similar HSJ1b expression levels were chosen for further study. The 19Q-HSJ1b $(n=444)$ and $128 \mathrm{Q}-\mathrm{HSJ} 1 \mathrm{~b}(n=388)$ transgenics were obtained by crossing HSJ1b transgenics with lines expressing stably integrated Pmec-3:btt57Q19: GFP or Pmec-3:htt57Q128:GFP. Animals expressing control (exon 1-19QGFP) were $50.1 \% \pm 2.4 \%$ touch sensitive at the tail, and animals expressing mutant transgene (exon 1-128Q-GFP) were $7 \% \pm 1.1 \%$ touch sensitive. Data are from at least 3 independent experiments performed in triplicate.

Aggregation was observed in all strains expressing the exon 1-128Q-GFP transgene. Images of worm mechanosensory neurons were captured from at least 100 animals for each strain over 5 different trials. Aggregation was measured as average gray value using MetaView software (Molecular Devices). Data are from at least 3 independent experiments performed in triplicate.

Cell extracts, subcellular fractionation, preparation of CCVs, and Western blots. Cell extracts were obtained by lysing cells 48 hours after transfection with NP40 lysis buffer $\left(2\right.$ minutes; $\left.4^{\circ} \mathrm{C}\right)$. The extracts were sonicated and centrifuged at $11,000 \mathrm{~g}\left(15\right.$ minutes; $\left.4^{\circ} \mathrm{C}\right)$. Proteins were loaded onto SDS-PAGE and subjected to Western blot analysis. For the experiment represented in Figure 2B, the samples were loaded without prior centrifugation. The aggregates remained in the stacking gel, whereas the soluble form migrated in the resolving gel. The aggregates were solubilized by formic acid treatment of the NP40 extracts (total) (31).

Subcellular fractionation was performed 48 hours after transfection as described previously (27). $T$ represents the total extract, and fractions P1, $\mathrm{P} 2$, S3, and P3 were obtained using differential centrifugations: P1, initial pellet of homogenate; P2, crude synaptosomes; P3 and S3, high-speed pellet and supernatant of $\mathrm{P} 2$, respectively.

CCVs were purified from transfected cells (67). Cells were homogenized in buffer A [0.1 M 2-(N-morpholino)ethane-sulfonic acid, pH 6.5; $1 \mathrm{mM}$ EGTA; $0.5 \mathrm{mM} \mathrm{MgCl}$; $0.83 \mathrm{mM}$ benzamidine; $0.23 \mathrm{mM}$ phenylmethylsulfonyl fluoride; $0.5 \mu \mathrm{g} / \mathrm{ml}$ aprotinin; and $0.5 \mu \mathrm{g} / \mathrm{ml}$ leupeptin]. The homogenate was centrifuged at $17,800 \mathrm{~g}$ for 20 minutes, and the supernatant was collected and centrifuged at 56,100 $\mathrm{g}$ for 1 hour. The pellet was resuspended in buffer $\mathrm{A}$, followed by dispersion through a 25 -gauge needle. The resuspended pellet was loaded on top of a solution containing $8 \%$ sucrose in buffer A and centrifuged for 2 hours at $115,800 \mathrm{~g}$. The supernatant (s) and the pellet containing the CCVs (p) were collected.

Antibodies. Antibodies used included: mouse monoclonal anti- $\beta$-actin AC15 (Sigma-Aldrich), anti-myc 9E10 (Calbiochem), anti-HA 16B12 (Covance Research Products Inc.), anti-clathrin, and anti-GM130 (BD Biosciences - Pharmingen); sheep polyclonal anti-HSJ1 (sHSJ1-277) (29); rabbit polyclonal anti-BDNF (Santa Cruz Biotechnology Inc.), anti-GFP
(Chemicon International), and anti-endoplasmic reticulum (68); human polyclonal anti-GMAP-210 (38).

Anti-mouse and anti-rabbit secondary antibodies conjugated to HRP were purchased from Jackson ImmunoResearch Laboratories Inc. Antimouse and anti-rabbit secondary antibodies conjugated to Alexa Fluor (A488, A555, and A630) were from Invitrogen Corp. Pictures of fixed cells were captured with a $3 \mathrm{D}$ deconvolution imaging system.

Analysis of $3 D$ images. The amount of clathrin-positive BDNF vesicles was quantified by measuring the colocalization between the 2 markers. Briefly, both clathrin and BDNF pictures were thresholded to segment vesicles and binarized. The percentage of colocalization was defined on binarized images as the ratio of clathrin-positive pixels overlapping BDNF-positive pixels to the total number of BDNF-positive pixels.

Quantification of Golgi area or vesicles that contained BDNF was achieved using the integrated morphometry analysis (IMA) from MetaMorph software version 6.2.6 (Molecular Devices). BDNF vesicles were defined by thresholding images to remove the background. Using an IMA size filter, objects ranging from 1-50 pixels ( $67 \mathrm{~nm}-3.35 \mu \mathrm{m}$ diameter) were considered as vesicles and scored (cytoplasmic BDNF vesicles), whereas the bigger perinuclear structure was considered to be Golgi apparatus. The associated signal (pixel intensity) was recorded and corresponds to the quantity of BDNF in the defined structures (BDNF content in Golgi or BDNF content in cytoplasmic vesicles). Data are from at least 3 independent experiments, with a minimum of 5 cells analyzed per condition.

BDNF immunoenzyme assays. BDNF assays were performed 48 hours after lipofection of neuronal cells. Cells were washed with PBS and incubated 30 minutes with DMEM alone or DMEM containing $100 \mu \mathrm{M}$ cystamine, and the supernatants were collected. For the time-course experiments, the incubation times with DMEM with or without cystamine were as stated. For BFA experiments, cells were first depolarized twice as previously described (27) in the absence or presence of BFA $(5 \mu \mathrm{M})$. Cells were then washed and treated for 30 minutes with DMEM or cystamine $(100 \mu \mathrm{M})$. The amount of BDNF was measured in supernatants and cell lysates using the BDNF Emax ImmunoAssay system (Promega). The data from transfected or treated cells are from at least 3 independent experiments performed in triplicate.

Animals. All experimental procedures were performed in strict accordance with the recommendations of the European Community (86/609/EEC) and the French National Committee (87/848) for care and use of laboratory animals. Live animal experiments were approved by the French Ethical Committee established by the Ministère de l'Agriculture et de la Forêt, Direction des Services Vétérinaires-Protection et Santé Animale (Evry, France).

R6/1 $\left(b d n f^{+/+} b t t^{m}\right)$ mice (46), BDNF heterozygous ( $b d n f^{\left.+/-b t t^{+/+}\right)}$mice (69), and $b d n f^{+/-} b t t^{m}$ double-mutant mice (26) were used in this study. All experiments were performed on male littermates from the $\mathrm{F}_{2}$ population to avoid strain and sex differences. Mice were housed together in numerical birth order in groups of mixed genotypes, and data were recorded for analysis by microchip mouse number. All experiments were conducted in a blinded manner with respect to genotype. Thirty minutes after the last administration of cysteamine (cysteamine bitartrate, or Cystagon [Orphan Europe], per os), 26- to 28 -week-old mice ( $n=4-5$ per condition) were deeply anesthetized in $\mathrm{CO}_{2}$ chamber, and their striata were dissected out on ice and rapidly frozen using $\mathrm{CO}_{2}$ pellets. Samples were then homogenized in lysis buffer (137 mM NaCl, 20 mM Tris-HCl, pH 8.0, 1\% Igepal, 10\% glycerol, $1 \mathrm{mM}$ PMSF, $10 \mu \mathrm{g} / \mathrm{ml}$ aprotinin, $1 \mu \mathrm{g} / \mathrm{ml}$ leupeptin), sonicated, and centrifuged $\left(10\right.$ minutes, $6,000 \mathrm{~g}$ at $\left.4^{\circ} \mathrm{C}\right)$. BDNF contents were determined in duplicate by the Emax ImmunoAssay system using $300 \mu \mathrm{g}$ of total protein for each point. C57BL/6 mice (males, age 5-6 weeks) were purchased from Charles River Laboratories. The $H d h^{109 Q}$ mice (males, age 15 weeks) have been previously described (45). Treatments with cystamine (Sigma-Aldrich; intra-peritoneal injections) or cysteamine started on day 1 with $100 \mathrm{mg} / \mathrm{kg}$ 
and were gradually increased to reach $300 \mathrm{mg} / \mathrm{kg}$ on day 7 (1 week). Mice were killed 30 minutes after the last administration. Blood was subjected to centrifugation (5 minutes; $2,700 \mathrm{~g}$ ), and supernatant (serum) was kept. BDNF levels were determined using $600 \mu \mathrm{g}$ of total protein (BDNF Emax ImmunoAssay system). A half frontal hemisphere of the brain was homogenized in NP40 lysis buffer and centrifuged 6,000 $\mathrm{g}\left(15\right.$ minutes; $\left.4^{\circ} \mathrm{C}\right)$, and $100 \mu \mathrm{g}$ of total protein of the supernatant was used to determine the amount of BDNF (BDNF Emax ImmunoAssay system).

Six-month-old male Sprague-Dawley rats $(n=4)$ weighing $550-580 \mathrm{~g}$ (Charles River Laboratories) were used for determination of the blood levels of BDNF at different time points before and after injection of either cysteamine (cysteamine dihydrochloride [Sigma-Aldrich]; $100 \mathrm{mg} / \mathrm{kg}$; intramuscular injection) or its vehicle (physiological saline solution). For blood sampling, animals were anesthetized by inhalation of a mixture of isoflurane (1\%), nitrous oxide (66\%), and oxygen (33\%). A catheter was inserted into and secured in the femoral vein. Blood samples $(\sim 300 \mu \mathrm{l})$ were collected every 15 minutes for 150 minutes.

Two long-tailed macaques (Macaca fascicularis) weighing 7.5 and $7 \mathrm{~kg}$ were treated for 14 months on a daily basis with slowly increasing doses of $3 \mathrm{NP}$ as previously described (49). At the time of blood collection, the $3 \mathrm{NP}$ dose was $30 \mathrm{mg} / \mathrm{kg} / \mathrm{d}$ given in 2 daily injections ( 1 injection at $9 \mathrm{am}$, 1 injection at $5 \mathrm{pm})$. These 2 animals had no overt striatal degeneration as assessed by magnetic resonance (MR) imaging, but based on localized proton MR spectroscopy examination, they showed reduced tricarboxylic acid cycle turnover and depletion in $\mathrm{N}$-acetyl-aspartate, glutamate, and GABA in the striatum. For blood sampling, the monkeys received a mixture of ketamine (15 mg/kg; Ketalar; PANPHARMA) and xylazine $(1.5 \mathrm{mg} / \mathrm{kg}$; Rompun; Bayer) before intubation. Animals were then anesthetized using an isoflurane/nitrous oxide mixture (1:66), controlled by an Ohmeda ventilator (Ohmeda OAV 7710; Datex-Ohmeda) with 33\% oxygen. The tidal volume was adjusted to achieve stable end-tidal $\mathrm{CO}_{2}$ tension between 38 and $40 \mathrm{mmHg}$.

After insertion of Teflon catheter into the saphena vein, blood samples $(500 \mu \mathrm{l})$ were collected every 15 minutes for 45 minutes before cysteamine injection. Cysteamine treatment (cysteamine hydrochloride [Sigma-Aldrich], $100 \mathrm{mg} / \mathrm{kg}$ ) was made by intramuscular injection in the hind limbs. Then blood samples were collected every 15 minutes for 120 minutes.

Immunohistochemistry. Cresyl violet staining and immunohistochemistry assays were performed on paraformaldehyde-fixed material as described previously (26). Administration of cysteamine or its vehicle was performed in 26-week-old mice ( $n=4-5$ per condition); 2 hours after the last administration, animals were deeply anesthetized and transcardially perfused with $4 \%$ paraformaldehyde solution in $0.1 \mathrm{M}$ sodium phosphate, $\mathrm{pH} 7.2$, at 15 days and 30 weeks of age. The brains were postfixed for 2 hours in the same solution, cryoprotected in increasing concentrations of sucrose/ PBS (10-30\%), and frozen in dry ice-cooled isopentane. Serial sections of $30 \mu \mathrm{m}$ depth were obtained every $0.3 \mathrm{~mm}$ and were processed for cresyl violet staining and immunohistochemistry.

Sections were processed as free-floating for immunohistochemistry. To block endogenous peroxidases, sections were incubated for 30 minutes with PBS containing $10 \%$ methanol and $3 \% \mathrm{H}_{2} \mathrm{O}_{2}$. Sections were washed 3 times in PBS and blocked for 1 hour with $2-10 \%$ normal serum in PBS. Tissue was then incubated with the anti-DARPP-32 antibody $(1: 10,000$; Chemicon International) in PBS containing 2\% normal goat serum for 16 hours at room temperature. Sections were washed 3 times and incubated with a biotinylated secondary antibody (1:200; Vector Laboratories) for 1-2 hours at room temperature in the same buffer as the primary antibody. The immunohistochemical reaction was developed using the VECTASTAIN $\mathrm{ABC}$ kit (Vector Laboratories). No signal was detected in controls that corresponded to the same treatment without the primary antibody.
Cell counts were performed in a blind manner with respect to genotype. Unbiased stereological counts of striatal cells were obtained from the entire neostriatum using Computer Assisted Stereology Toolbox (CAST) software (Olympus). The dissector counting method was employed to analyze coronal sections spaced $300 \mu \mathrm{m}$ apart. The counting frames were randomly sampled. We counted the neuron-like cells in cresyl violet staining as the cells with a large nonpyknotic nucleus with clear nucleoli.

Quantification of DARPP-32-positive cells in the striatum of 3-4 animals per condition revealed significant differences (ANOVA, $F_{5,15}=8.98$; $P<0.0004[P<0.05$ was considered statistically significant $]$ ). There was a significant difference in the number of DARPP-32-positive cells per striatum between $b d n f^{+/+} b t t^{w t}$ mice and $b d n f^{+/+} b t t^{m}$ mice (post-hoc Fisher's test, $P=0.003)$ and between $b d n f^{+/+} b t t^{w t}$ mice and $b d n f^{+/-h t t^{m}}$ mice with (post-hoc Fisher's test, $P=0.00022$ ) or without cysteamine treatment (post-hoc Fisher's test, $P=0.00043$ ). Cysteamine induced a significant increase in the number of DARPP-32-positive cells per striatum in $b d n f^{+/+} b t t^{m}$ mice (post-hoc Fisher's test, $P=0.048$ ) but not in $b d n f^{+/-} b t t^{m}$ mice (post-hoc Fisher's test, NS).

In situ bybridization. Administration of cysteamine or its vehicle was performed in 17 - to 18 -week-old mice $(n=3-4)$. After 1 week, animals were killed 2 hours after the last administration, and brains were dissected out and frozen in dry-ice cooled isopentane. Sections were processed for in situ hybridization with radioactive oligonucleotide probes for preproenkephalin (PPE) as previously described (70). The slides were exposed to Biomax MR (Kodak) for 7 days. The expression of PPE was quantified on in situ hybridization films. Consecutive sections (18-20 sections/animal) were scanned, and mRNA levels were analyzed using the ImageJ program (http://rsb.info.nih.gov/ij/). The striatal area was determined according to anatomical landmarks, and the intensity was quantified. The background signal of the same adjacent area outside of the brain was subtracted.

Quantification of ENK mRNA levels in 3-4 animals per each condition revealed significant differences (ANOVA, $F_{7,16}=17.71 ; P=0.000002$ ). There was a significant difference in $E N K$ mRNA levels between $b d n f^{+/+} h t t^{w t}$ mice and both $b d n f^{++} b t t^{m}$ mice (post-hoc Fisher's test, $P=0.0084$ ) and $b d n f^{+/-} b t t^{m}$ mice (post-hoc Fisher's test, $P=0.000001$ ); and between $b d n f^{+/+} b t t^{m}$ mice and $b d n f^{+/-} b t t^{m}$ mice (post-hoc Fisher's test, $\left.P=0.0024\right)$. Cysteamine treatment induced an increase in ENK mRNA levels in $b d n f^{+/+} b t t^{m}$ mice (post-hoc Fisher's test, $P=0.057$ ) and in $b d n f^{+/-h t t^{m}}$ mice (post-hoc Fisher's test, $P=0.0002$ ).

\section{Acknowledgments}

We greatly acknowledge V.C. Wheeler and M.E. MacDonald for Hdh ${ }^{109 Q}$ mice; G.V. Johnson and H.Y. Zoghbi for constructs; Ana López for her help in mice care; E. Coudrier and M. Bornens for antibodies; the Institut Curie Imaging Facility; the Huntington French Speaking Group for valuable discussions; S. Julien-Grille, H. Medyouf, and members of the Saudou/Humbert laboratory for help and comments. We gratefully acknowledge Harvard Brain Tissue Resource Center, supported in part by Public Health Service grant number MH/NS 31862, for providing human brain tissue. This research was supported by the Association pour la Recherche sur le Cancer (ARC 3665, to S. Humbert), Fondation pour la Recherche Médicale and Fondation BNP Paribas (to F. Saudou), HighQ Foundation (to J.A. Parker, C. Néri, F. Saudou, and S. Humbert), Association Française contre les Myopathies (AFM; to F. Saudou), Provital - P. Chevalier (to F. Saudou and S. Humbert), INSERM (to C. Néri), the Ministerio de Educación y Ciencia (to J. Alberch and J.M. 
Canals), and Fundació La Caixa (to J. Alberch). J.R. Pineda is a fellow of the Ministerio de Educación y Ciencia (Spain). M. Borrell-Pagès is supported by an EMBO long-term fellowship. F. Saudou is a recipient of an EMBO Young Investigator award and is an INSERM/Assistance Publique - Hôpitaux de Paris investigator. S. Humbert is an INSERM investigator.

Received for publication December 7, 2005, and accepted in revised form February 14, 2006.
Address correspondence to: F. Saudou and S. Humbert, Institut Curie - UMR146 CNRS, Bat. 110, Centre Universitaire, Orsay, 91400, France. Phone: 33-169-86-30-24; Fax: 33-169-07-45-25; E-mail: frederic.saudou@curie.u-psud.fr (F. Saudou). Phone: 33169-86-30-69; Fax: 33-169-07-45-25; E-mail: sandrine.humbert@ curie.u-psud.fr (S. Humbert).

Josep M. Canals, Fabrice P. Cordelières, and J. Alex Parker contributed equally to this work.
1. Young, A.B. 2003. Huntingtin in health and disease. J. Clin. Invest. 111:299-302. doi:10.1172/ JCI200317742.

2. MacDonald, M.E., Gines, S., Gusella, J.F., and Wheeler, V.C. 2003. Huntington's disease. Neuromolecular Med. 4:7-20.

3. Melino, G., and Piacentini, M. 1998. 'Tissue' transglutaminase in cell death: a downstream or a multifunctional upstream effector? FEBS Lett. 430:59-63.

4. Lesort, M., Tucholski, J., Miller, M.L., and Johnson, G.V. 2000. Tissue transglutaminase: a possible role in neurodegenerative diseases. Prog. Neurobiol. 61:439-463.

5. Green, H. 1993. Human genetic diseases due to codon reiteration: relationship to an evolutionary mechanism. Cell. 74:955-956.

6. Cooper, A.J.L., et al. 1997. Polyglutamine domains are substrates of tissue transglutaminase - does transglutaminase play a role in expanded Cag/ Poly-Q neurodegenerative diseases? J. Neurochem. 69:431-434.

7. Kahlem, P., Green, H., and Djian, P. 1998. Transglutaminase action imitates Huntington's disease: selective polymerization of huntingtin containing expanded polyglutamine. Mol. Cell. 1:595-601.

8. Karpuj, M.V., et al. 1999. Transglutaminase aggregates huntingtin into nonamyloidogenic polymers, and its enzymatic activity increases in Huntington's disease brain nuclei. Proc. Natl. Acad. Sci. U. S. A. 96:7388-7393

9. Lesort, M., Chun, W., Johnson, G.V., and Ferrante, R.J. 1999. Tissue transglutaminase is increased in Huntington's disease brain. J. Neurochem. 73:2018-2027.

10. Dedeoglu, A., et al. 2002. Therapeutic effects of cystamine in a murine model of Huntington's disease. J. Neurosci. 22:8942-8950.

11. Karpuj, M.V., et al. 2002. Prolonged survival and decreased abnormal movements in transgenic model of Huntington disease, with administration of the transglutaminase inhibitor cystamine. Nat. Med. 8:143-149.

12. Zainelli, G.M., Ross, C.A., Troncoso, J.C., and Muma, N.A. 2003. Transglutaminase cross-links in intranuclear inclusions in Huntington disease. J. Neuropathol. Exp. Neurol. 62:14-24.

13. Mastroberardino, P.G., et al. 2002. 'Tissue' transglutaminase ablation reduces neuronal death and prolongs survival in a mouse model of Huntington's disease. Cell Death Differ. 9:873-880.

14. Bailey, C.D., and Johnson, G.V. 2005. Tissue transglutaminase contributes to disease progression in the R6/2 Huntington's disease mouse model via aggregate-independent mechanisms. J. Neurochem. 92:83-92.

15. Wang, X., et al. 2005. Cerebral PET imaging and histological evidence of transglutaminase inhibitor cystamine induced neuroprotection in transgenic R6/2 mouse model of Huntington's disease. J. Newrol. Sci. 231:57-66.

16. Bailey, C.D., and Johnson, G.V. 2005. The protective effects of cystamine in the R6/2 Huntington's disease mouse involve mechanisms other than the inhibition of tissue transglutaminase. Neurobiol. Aging. doi:10.1016/j.neurobiolaging.2005.04.001.

17. Pinto, J.T., et al. 2005. Treatment of YAC128 mice and their wild-type littermates with cystamine does not lead to its accumulation in plasma or brain: implications for the treatment of Huntington disease. J. Neurochem. 94:1087-1101.

18. Lesort, M., Lee, M., Tucholski, J., and Johnson, G.V. 2003. Cystamine inhibits caspase activity. Implications for the treatment of polyglutamine disorders. J. Biol. Chem. 278:3825-3830.

19. Fox, J.H., et al. 2004. Cystamine increases L-cysteine levels in Huntington's disease transgenic mouse brain and in a PC12 model of polyglutamine aggregation. J. Neurochem. 91:413-422.

20. Opal, P., and Zoghbi, H.Y. 2002. The role of chaperones in polyglutamine disease. Trends Mol. Med. 8:232-236.

21. Bonini, N.M. 2002. Chaperoning brain degeneration. Proc. Natl. Acad. Sci. U. S. A. 99:16407-16411.

22. Muchowski, P.J., and Wacker, J.L. 2005. Modulation of neurodegeneration by molecular chaperones. Nat. Rev. Neurosci. 6:11-22.

23. Sherman, M.Y., and Goldberg, A.L. 2001. Cellular defenses against unfolded proteins: a cell biologist thinks about neurodegenerative diseases. Neuron. 29:15-32.

24. Saudou, F., Finkbeiner, S., Devys, D., and Greenberg, M.E. 1998. Huntingtin acts in the nucleus to induce apoptosis but death does not correlate with the formation of intranuclear inclusions. Cell. 95:55-66.

25. Zuccato, C., et al. 2001. Loss of huntingtin-mediated BDNF gene transcription in Huntington's disease. Science. 293:493-498.

26. Canals, J.M., et al. 2004. Brain-derived neurotrophic factor regulates the onset and severity of motor dysfunction associated with enkephalinergic neuronal degeneration in Huntington's disease. J. Neurosci. 24:7727-7739.

27. Gauthier, L.R., et al. 2004. Huntingtin controls neurotrophic support and survival of neurons by enhancing BDNF vesicular transport along microtubules. Cell. 118:127-138.

28. Trettel, F., et al. 2000. Dominant phenotypes produced by the HD mutation in STHdh(Q111) striatal cells. Hum. Mol. Genet. 9:2799-2809.

29. Chapple, J.P., and Cheetham, M.E. 2003. The chaperone environment at the cytoplasmic face of the endoplasmic reticulum can modulate rhodopsin processing and inclusion formation. J. Biol. Chem. 278:19087-19094.

30. Sakahira, H., Breuer, P., Hayer-Hartl, M.K., and Hartl, F.U. 2002. Molecular chaperones as modulators of polyglutamine protein aggregation and toxicity. Proc. Natl. Acad. Sci. U. S. A. 99:16412-16418.

31. Hazeki, N., Tukamoto, T., Goto, J., and Kanazawa, I. 2000. Formic acid dissolves aggregates of an $\mathrm{N}$-terminal huntingtin fragment containing an expanded polyglutamine tract: applying to quantification of protein components of the aggregates. Biochem. Biophys. Res. Commun. 277:386-393.

32. Parker, J.A., et al. 2001. Expanded polyglutamines in Caenorhabditis elegans cause axonal abnormalities and severe dysfunction of PLM mechanosensory neurons without cell death. Proc. Natl. Acad. Sci. U. S. A. 98:13318-13323.

33. Parker, J.A., et al. 2005. Resveratrol rescues mutant polyglutamine cytotoxicity in nematode and mammalian neurons. Nat. Genet. 37:349-350.

34. Cheetham, M.E., Jackson, A.P., and Anderton, B.H 1994. Regulation of 70-kDa heat-shock-protein ATPase activity and substrate binding by human DNAJ-like proteins, HSJ1a and HSJ1b. Eur. J. Biochem. 226:99-107.

35. Cheetham, M.E., Anderton, B.H., and Jackson, A.P. 1996. Inhibition of hsc70-catalysed clathrin uncoating by HSJ1 proteins. Biochem. J. 319:103-108.

36. Harjes, P., and Wanker, E.E. 2003. The hunt for huntingtin function: interaction partners tell many different stories. Trends Biochem. Sci. 28:425-433.

37. Gleeson, P.A., Lock, J.G., Luke, M.R., and Stow, J.L. 2004. Domains of the TGN: coats, tethers and G proteins. Traffic. 5:315-326.

38. Infante, C., Ramos-Morales, F., Fedriani, C., Bornens, M., and Rios, R.M. 1999. GMAP-210, a cis-Golgi network-associated protein, is a minus end microtubule-binding protein. J. Cell Biol. 145:83-98.

39. Klausner, R.D., Donaldson, J.G., and LippincottSchwartz, J. 1992. Brefeldin A: insights into the control of membrane traffic and organelle structure. J. Cell Biol. 116:1071-1080.

40. Campisi, A., et al. 2003. Glutamate-induced increases in transglutaminase activity in primary cultures of astroglial cells. Brain Res. 978:24-30.

41. Pastuszko, A., Wilson, D.F., and Erecinska, M. 1986. A role for transglutaminase in neurotransmitter release by rat brain synaptosomes. J. Neurochem. 46:499-508.

42. Gahl, W.A., Thoene, J.G., and Schneider, J.A. 2002. Cystinosis. N. Engl. J. Med. 347:111-121.

43. Shults, C., et al. 1986. Huntington's disease: effect of cysteamine, a somatostatin-depleting agent. Neurology. 36:1099-1102.

44. Dubinsky, R., and Gray, C. 2005. CYTE-I-HD: phase I dose finding and tolerability study of cysteamine (Cystagon) in Huntington's disease. Mov. Disord. doi: $10.1002 / \mathrm{mds} .20756$.

45. Wheeler, V.C., et al. 2000. Long glutamine tracts cause nuclear localization of a novel form of huntingtin in medium spiny striatal neurons in HdhQ92 and HdhQ111 knock- in mice. Hum. Mol. Genet. 9:503-513.

46. Mangiarini, L., et al. 1996. Exon 1 of the HD gene with an expanded CAG repeat is sufficient to cause a progressive neurological phenotype in transgenic mice. Cell. 87:493-506.

47. Wheeler, V.C., et al. 2002. Early phenotypes that presage late-onset neurodegenerative disease allow testing of modifiers in Hdh CAG knock-in mice. Hum. Mol. Genet. 11:633-640.

48. Brouillet, E., et al. 1995. Chronic mitochondrial energy impairment produces selective striatal degeneration and abnormal choreiform movements in primates. Proc. Natl. Acad. Sci. U. S. A. 92:7105-7109.

49. Palfi, S., et al. 1998. Fetal striatal allografts reverse cognitive deficits in a primate model of Huntington disease. Nat. Med. 4:963-966.

50. Petrova, P., et al. 2003. MANF: a new mesencephalic, astrocyte-derived neurotrophic factor with selectivity for dopaminergic neurons. J. Mol. Neurosci. 20:173-188. 
51. Legendre-Guillemin, V., et al. 2005. Huntingtin interacting protein 1 (HIP1) regulates clathrin assembly through direct binding to the regulatory region of the clathrin light chain. J. Biol. Chem. 280:6101-6108.

52. Chen, C.Y., and Brodsky, F.M. 2005. Huntingtininteracting protein 1 (Hip1) and Hip1-related protein (Hip1R) bind the conserved sequence of clathrin light chains and thereby influence clathrin assembly in vitro and actin distribution in vivo. J. Biol. Chem. 280:6109-6117.

53. Bungay, P.J., Potter, J.M., and Griffin, M. 1984. The inhibition of glucose-stimulated insulin secretion by primary amines. A role for transglutaminase in the secretory mechanism. Biochem. J. 219:819-827.

54. Gobbi, M., Frittoli, E., and Mennini, T. 1996. Role of transglutaminase in [3H]5-HT release from synaptosomes and in the inhibitory effect of tetanus toxin. Neurochem. Int. 29:129-134.

55. Davies, P.J., et al. 1984. Studies on the effects of dansylcadaverine and related compounds on receptor-mediated endocytosis in cultured cells. Diabetes Care. 7:35-41.

56. Howe, C.L., Valletta, J.S., Rusnak, A.S., and Mobley, W.C. 2001. NGF signaling from clathrin-coated vesicles: evidence that signaling endosomes serve as a platform for the Ras-MAPK pathway. Neuron. 32:801-814
57. Jeitner, T.M., Delikatny, E.J., Ahlqvist, J., Capper, H., and Cooper, A.J. 2005. Mechanism for the inhibition of transglutaminase 2 by cystamine. Biochem. Pharmacol. 69:961-970.

58. Tenneze, L., Daurat, V., Tibi, A., Chaumet-Riffaud, P., and Funck-Brentano, C. 1999. A study of the relative bioavailability of cysteamine hydrochloride, cysteamine bitartrate and phosphocysteamine in healthy adult male volunteers. Br. J. Clin. Pharmacol. 47:49-52.

59. Belldina, E.B., Huang, M.Y., Schneider, J.A., Brundage, R.C., and Tracy, T.S. 2003. Steady-state pharmacokinetics and pharmacodynamics of cysteamine bitartrate in paediatric nephropathic cystinosis patients. Br. J. Clin. Pharmacol. 56:520-525.

60. Douma, S., et al. 2004. Suppression of anoikis and induction of metastasis by the neurotrophic receptor TrkB. Nature. 430:1034-1039.

61. Kleta, R., et al. 2004. Long-term follow-up of welltreated nephropathic cystinosis patients. J. Pediatr. 145:555-560.

62. Chun, W., et al. 2001. Tissue transglutaminase selectively modifies proteins associated with truncated mutant huntingtin in intact cells. Neurobiol. Dis. 8:391-404.

63. Cummings, C.J., et al. 1998. Chaperone suppression of aggregation and altered subcellular proteasome localization imply protein misfolding in SCA1. Nat.
Genet. 19:148-154.

64. Haubensak, W., Narz, F., Heumann, R., and Lessmann, V. 1998. BDNF-GFP containing secretory granules are localized in the vicinity of synaptic junctions of cultured cortical neurons. J. Cell Sci. 111:1483-1493.

65. Humbert, S., et al. 2002. The IGF-1/Akt pathway is neuroprotective in Huntington's disease and involves Huntingtin phosphorylation by Akt. Dev. Cell. 2:831-837.

66. Brenner, S. 1974. The genetics of Caenorhabditis elegans. Genetics. 77:71-94

67. Metzler, M., et al. 2001. HIP1 functions in clathrin-mediated endocytosis through binding to clathrin and adaptor protein 2. J. Biol. Chem. 276:39271-39276.

68. Louvard, D., Reggio, H., and Warren, G. 1982. Antibodies to the Golgi complex and the rough endoplasmic reticulum. J. Cell Biol. 92:92-107.

69. Ernfors, P., Lee, K.F., and Jaenisch, R. 1994. Mice lacking brain-derived neurotrophic factor develop with sensory deficits. Nature. 368:147-150.

70. Perez-Navarro, E., Alberch, J., Neveu, I., and Arenas, E. 1999. Brain-derived neurotrophic factor, neurotrophin- 3 and neurotrophin- $4 / 5$ differentially regulate the phenotype and prevent degenerative changes in striatal projection neurons after excitotoxicity in vivo. Neuroscience. 91:1257-1264. 